\title{
Genomic characterization, phylogenetic analysis, and identification of virulence factors in Aerococcus sanguinicola and Aerococcus urinae strains isolated from infection episodes
}

Carkaci, Derya; Højholt, Katrine; Nielsen, Xiaohui Chen; Dargis, Rimtas; Rasmussen, Simon; Skovgaard, Ole; Fuursted, Kurt; Andersen, Paal Skytt; Stegger, Marc; Christensen, Jens Jørgen

Published in:

Microbial Pathogenesis

Link to article, DOI:

10.1016/j.micpath.2017.09.042

Publication date:

2017

Document Version

Peer reviewed version

Link back to DTU Orbit

Citation (APA):

Carkaci, D., Højholt, K., Nielsen, X. C., Dargis, R., Rasmussen, S., Skovgaard, O., Fuursted, K., Andersen, P. S., Stegger, M., \& Christensen, J. J. (2017). Genomic characterization, phylogenetic analysis, and identification of virulence factors in Aerococcus sanguinicola and Aerococcus urinae strains isolated from infection episodes. Microbial Pathogenesis, 112, 327-340. https://doi.org/10.1016/j.micpath.2017.09.042

\section{General rights}

Copyright and moral rights for the publications made accessible in the public portal are retained by the authors and/or other copyright owners and it is a condition of accessing publications that users recognise and abide by the legal requirements associated with these rights.

- Users may download and print one copy of any publication from the public portal for the purpose of private study or research.

- You may not further distribute the material or use it for any profit-making activity or commercial gain

- You may freely distribute the URL identifying the publication in the public portal 
TITLE

Genomic Characterization, Phylogenetic Analysis, and Identification of Virulence Factors in Aerococcus sanguinicola and Aerococcus urinae Strains Isolated from Infection Episodes

4

5

6

7

\section{AUTHOR NAMES}

Derya Carkaci ${ }^{1,2,3,}{ }^{,}$, Katrine $H \varnothing j$ jholt ${ }^{1,4,}{ }^{*}$, Xiaohui Chen Nielsen ${ }^{1}$, Rimtas Dargis $^{1}$, Simon Rasmussen ${ }^{4}$, Ole Skovgaard $^{2}$, Kurt Fuursted ${ }^{3}$, Paal Skytt Andersen ${ }^{3,5}$, Marc Stegger ${ }^{3}$ \& Jens Jørgen Christensen ${ }^{1,6}$.

* Shared authorship.

Derya Carkaci (DC)

Katrine $\mathrm{H} \varnothing$ jholt $(\mathrm{KH})$

Xiaohui Chen Nielsen (XCN)

Rimtas Dargis (RD)

Simon Rasmussen (SR)

Ole Skovgaard (OS)

Kurt Fuursted (KF)

Paal Skytt Andersen (PSA)

Marc Stegger (MS)

Jens Jørgen Christensen (JJC) derya.carkaci@gmail.com

katrine.hojholt@bioinformatics.dtu.dk

xcn@regionsjaelland.dk

rida@regionsjaelland.dk

simon@bioinformatics.dtu.dk

olesk@ruc.dk

kfu@ssi.dk

psa@ssi.dk

mtg@ssi.dk

jejc@regionsjaelland.dk

\section{AFFILIATION}

${ }^{1}$ Department of Clinical Microbiology, Slagelse Hospital, Slagelse, Denmark.

${ }^{2}$ Department of Science and Environment, Roskilde University, Roskilde, Denmark.

${ }^{3}$ Department of Microbiology \& Infection Control, Statens Serum Institut, Copenhagen, Denmark.

${ }^{4}$ Department of Bio and Health Informatics, Technical University of Denmark, Kongens Lyngby, Denmark

${ }^{5}$ Department of Veterinary Disease Biology, Faculty of Health and Medical Sciences, University of Copenhagen,

Copenhagen, Denmark.

${ }^{6}$ Department of Clinical Medicine, University of Copenhagen, Copenhagen, Denmark.

\section{CORRESPONDING AUTHOR INFORMATION}

Address correspondence to: Jens Jørgen Christensen, Department of Clinical Microbiology, Slagelse Hospital, Ingemannsvej 46, DK-4200 Slagelse, Denmark. Email: jejc@regionsjaelland.dk. 
39

40

41

42

43 44 45

46

47 48

\section{HIGHLIGHTS}

Bacterial adhesion gene homologs were identified in $A$. sanguinicola ( $h t p B, f b p A, I m b$, and ilpA) and $A$. urinae ( htpB, lap, Imb, fbp54, and ilpA) genomes.

Capsular polysaccharide (CPS) gene homologs were identified in A. sanguinicola (15 genes) and A. urinae (1116 genes) strains, giving rise to one and five types of putative CPS loci, respectively.

Marked differences were observed within A. urinae 1984-2004 and 2010-2015 strains in regards to genome sizes, core-genomes, proteome conservations, and phylogenetic analysis. 


\section{ABSTRACT}

Aerococcus sanguinicola and Aerococcus urinae are emerging pathogens in clinical settings mostly being causative agents of urinary tract infections (UTIS), urogenic sepsis and more seldomly complicated infective endocarditis (IE). Limited knowledge exists concerning the pathogenicity of these two species. Eight clinical A. sanguinicola (isolated from 2009-2015) and 40 clinical A. urinae (isolated from 1984-2015) strains from episodes of UTIs, bacteremia, and IE were whole-genome sequenced (WGS) to analyze genomic diversity and characterization of virulence genes involved in the bacterial pathogenicity.

A. sanguinicola genome sizes were 2.06-2.12 Mb with a 47.4-47.6 \% GC-contents, and 1,783-1,905 genes were predicted whereof 1,170 were core-genes. In case of $A$. urinae strains, the genome sizes were 1.932.44 Mb with 41.6-42.6\% GC-contents, and 1,708-2,256 genes of which 907 were core-genes.

Marked differences were observed within A. urinae strains with respect to the average genome sizes, number and sequence identity of core-genes, proteome conservations, phylogenetic analysis, and putative capsular polysaccharide (CPS) loci sequences. Strains of A. sanguinicola showed high degree of homology. Phylogenetic analyses showed the $40 \mathrm{~A}$. urinae strains formed two clusters according to two time periods: 1984-2004 strains and 2010-2015 strains.

Genes that were homologs to virulence genes associated with bacterial adhesion and antiphagocytosis were identified by aligning $A$. sanguinicola and $A$. urinae pan- and core-genes against Virulence Factors of Bacterial Pathogens (VFDB). Bacterial adherence associated gene homologs were present in genomes of $A$. sanguinicola ( $h t p B, f b p A, I m b$, and $i l p A)$ and $A$. urinae ( $h t p B$, lap, Imb, fbp54, and ilpA). Fifteen and 11-16 CPS gene homologs were identified in genomes of $A$. sanguinicola and $A$. urinae strains, respectively. Analysis of these genes identified one type of putative CPS locus within all A. sanguinicola strains. In $A$. urinae genomes, five different CPS loci types were identified with variations in CPS locus sizes, genetic content, and structural organization.

In conclusion, this is the first study dealing with WGS and comparative genomics of clinical $A$. sanguinicola and A. urinae strains from episodes of UTIs, bacteremia, and IE. Gene homologs associated with antiphagocytosis and bacterial adherence were identified and genetic variability was observed within $A$. urinae genomes. These findings contributes with important knowledge and basis for future molecular and experimental pathogenicity study of UTIs, bacteremia, and IE causing A. sanguinicola and A. urinae strains.

\section{KEYWORDS}

Aerococcus sanguinicola; Aerococcus urinae; Infective endocarditis; Urinary tract infections; Capsular Polysaccharide; Bacterial adherence. 


\section{INTRODUCTION}

The genus Aerococcus was first described in 1953 and consists nowadays of eight species of which Aerococcus viridans for a long time was the only species within the genus [1,2].

Aerococcus urinae was isolated in 1984 from a urine sample from a patient with verified urinary tract infection (UTI). This strain was characterized in 1989 as an Aerococcus-like organism and reclassified into its own species designation in 1992 [3,4]. Aerococcus sanguinicola was isolated in 1999 from an infective endocarditis (IE) suspected patient and in 2001 designated into its own species [5]. Both species are associated with UTIs worldwide, especially in elderly patients with predisposing conditions $[6,7]$.

The prevalence of $A$. urinae in urine samples vary from $0.25 \%$ to $4 \%[7,8]$. Both species were isolated from blood of patients suffering from urogenic sepsis, in few cases from patients with complicating IE and casuistically isolated from other foci [9]. Recognition of both species may be limited by their fastidious growth, often requiring supplementation with $\mathrm{CO}_{2}$ for optimal growth $[6,10]$. Aerococci share colony morphology with $\alpha$-hemolytic streptococci and have a microscopic appearance similar to staphylococci, which adds to the risk of misinterpretation and misidentification [9]. At present, very limited knowledge exists regarding the bacterial pathogenicity and virulence mechanisms that lead to and maintain infections.

In clinical microbiology laboratories, diagnosing $A$. urinae and $A$. sanguinicola infections have been challenging [9]. Matrix-assisted laser desorption ionization time-of-flight mass spectrometry (MALDI-TOF MS), however, identifies both species rapidly and accurately, allowing clinical laboratories to correctly identify strains with increasing frequency of detection [11,12]. The species identifications can also be achieved with analysis of the 16S rRNA gene sequence [13] or the 16S-23S rRNA Intergenic Spacer Region [14].

Bacterial adherence and invasion to host tissue and cells increases the bacterial pathogenicity in infectious diseases as UTI [15] and IE [16]. Several host cell surface molecules are involved in the adhesive process in other pathogenic species, including fibronectin-binding proteins of Streptococcus pyogenes (fbp54) [17] and Listeria monocytogenes (fbpA) [18], laminin-binding protein of Streptococcus agalactiae (Imb) [19], and the Listeria adhesion protein (Iap) [20]. A study from Shannon et al. (2010) described for the first time biofilm formation and stimulated biofilm production of $A$. urinae during exposure to human plasma [21]. The same study showed activation and aggregation of human platelets by A. urinae. Similarly, Senneby et al. (2014) demonstrated biofilm production in A. sanguinicola strains [22].

Expression of capsular polysaccharide (CPS) facilitates bacterial protection against host immune phagocytosis [23]. Within genus Aerococcus, CPS expression were reported in a variant of A. viridans, A. viridans var. homari, which is a lobster pathogen causing gaffkemia [24]. The same study group showed upregulated 
115

expression of molecular heat shock protein 60 (Hsp60) in virulent A. viridans strains compared to an avirulent strain [25].

A study from Christensen et al. showed genetic heterogeneity within a group of $A$. urinae strains. Fourteen Danish strains from 1984 to 1994 constituted a homogeneous group compared to seven heterogeneous non-Danish strains from 1985 to 1995 using DNA hybridization and phenotypic analysis [26].

Application of WGS has drastically expanded the understanding of the microbial world. The availability of bacterial genome data enables comprehensive bacterial comparisons and provides a better understanding of genome structures, evolutionary diversity, pathogenicity, and antimicrobial resistance [27]. In order to obtain further understanding of the genetic context of genes and to have a suitable high quality reference strain for the comparative genomics, complete and closed genomes of six Aerococcus type strains were recently achieved [28].

No whole-genome comparisons and genomic characterizations of $A$. urinae and $A$. sanguinicola have previously been performed. The aim of this study was to investigate the genomes of $40 \mathrm{~A}$. urinae and eight $A$. sanguinicola strains in order to gain insight into their pan- and core-genome content and to identify putative virulence mechanisms that may be associated with human disease. Moreover, we compared WGS data and inferred phylogenetic relationships of the 40 clinical $A$. urinae strains from two different time periods of 1984-2004 and 2010-2015, to analyze if the genomic diversity may be specific for the time period of strain isolations and type of infections.

\section{MATERIALS AND METHODS}

2.1. Bacterial strain characteristics, identifications, DNA isolation, genome sequencing, and verification of species identifications

\subsubsection{Bacterial strains and species level identifications.}

Eight clinical A. sanguinicola strains were collected between 2009 and 2015. Four isolates from two patients (one urine and one blood isolate for each patient), two isolates from two patients (one urine and one blood isolate), and two urine isolates from one patient (Supplementary material A).

Forty clinical A. urinae strains were collected from 32 patients between 1984 and 2015, twenty of these strains from 1984-2004 and the remaining 20 strains from 2010-2015. Twenty-four strains were isolated from 24 individual patients: From urine samples of UTI verified patients $(n=9)$, from positive blood cultures of patients with bacteremia $(n=9)$ and with verified IE $(n=6)$. Fourteen strains were isolated from seven patients, both from urine $(n=7)$ and blood culture $(n=7)$ of each patient (paired strains). Two strains were 
146 isolated as a pair from one patient, one blood isolate and one post mortem heart valve sample 147 (Supplementary material A).

148 All strains were received from departments of clinical microbiology in Denmark. Identification to the species 149 level was accomplished using MALDI-TOF MS v4.0.0.1 (5627 reference entries) (Bruker Daltonics, Germany) 150 with a score above 2.0 at the Department of Clinical Microbiology, Slagelse Hospital, Denmark. Clinical 151 strains were stored at $-80^{\circ} \mathrm{C}$ in bovine broth with $10 \%$ glycerol (SSI Diagnostica, Denmark) until use.

152 Type strains of A. sanguinicola CCUG $43001^{\top}$ and A. urinae CCUG $36881^{\top}$ were obtained from the Culture 153 Collection, University of Göteborg (www.ccug.se) and used as reference strains for the comparative genomic 154 analyses. A. sanguinicola CCUG $43001^{\top}$ (isolated in 2001) and A. urinae CCUG $36881^{\top}$ (isolated in 1984, 155 characterized in 1989, and reclassified in 1992) were isolated from a positive blood culture from a patient 156 having bacteremia and from urine sample of a patient having UTIs, respectively [28].

157 The bacterial species identification and strain characteristics were denominated in a three-part identifier, 158 such as "Au-01-U13". The initial two letter refers to the species identification (As for A. sanguinicola and Au 159 for $A$. urinae), followed by a strain specific number. The final three characters describe the source of 160 isolation (blood $(B)$, urine $(U)$ or heart valve $(H)$ ), and the year of strain isolation. "Au-01-U13" is a strain of $A$. 161 urinae from a positive urine sample which was isolated in 2013.

162 Numbering of the paired A. sanguinicola strains, pair no. 1) As-24-U13 \& As-25-U14, 2) As-41-B14 \& As-46$163 \mathrm{U14}$, and 3) As-55-B15 \& As-56-U15. Numbering of the paired A. urinae strains, pair no. 1) Au-02-B96 \& Au164 03-U96, 2) Au-44-B14 \& Au-47-U14, 3) Au-49-B14 \& Au-50-U14, 4) Au-51-B15 \& Au-52-U15, 5) Au-53-B14 \& Au-54-U14, 6) Au-57-B15 \& Au-58-U15, 7) Au-59-B15 \& Au-60-U15, and 8) Au-18-B93 \& Au-19-H93.

Genomes of A. urinae CCUG 36881 ${ }^{\top}$ (CP014161), A. urinae ACS-120-V-Col10a (CP002512), and A. urinae AU3 (LUKP00000000.1) strains were obtained from NCBI GenBank for comparative analyses. A. urinae CCUG $36881^{\top}$ was isolated from a positive human urine of a UTI infected person in 1984. A. urinae ACS-120-VCol10a was isolated from a human vagina sample in Belgium in 2007. A. urinae AU3 was isolated from the human blood of a patient with bacteremia in Sweden in 2010.

\subsubsection{DNA isolation and extraction.}

Strains were maintained by no more than three-to-four serial overnight passages at $35-37^{\circ} \mathrm{C}$ in ambient air with $5 \% \mathrm{CO}_{2}$ enrichment on $5 \%$ blood agar plates (SSI Diagnostica, Denmark). Extraction of genomic DNA was carried out at Department of Microbiology and Infection Control, Statens Serum Institut, Denmark using the DNeasy Blood \& Tissue kit, as described by the manufacturer (Qiagen, Denmark). Extraction of genomic DNA and WGS of A. sanguinicola CCUG $43001^{\top}$ and A. urinae CCUG $36881^{\top}$ were described in Carkaci et al. [28]. 
179

180

181

182

183

184

185

186

187

188

189

190

191

192

193

194

195

196

197

198

199

200

201

202

203

204

205

206

207

208

209

210

\subsubsection{Genome sequencing and pre-processing of sequence data.}

Fragment libraries were constructed using the Nextera XT DNA Sample Preparation Kit (Illumina, USA) followed by 251-bp or 150-bp paired-end sequencing on MiSeq or NextSeq sequencers (Illumina, USA), respectively, according to manufacturer's instructions. The Illumina demultiplexing process removed adapter sequences.

Quality of sequence reads were validated using FastQC v0.11.2 [29] and filtered using PRINSEQ v0.20.4 [30]. High-quality sequence reads were de novo assembled using SPAdes v3.6.0 [31] with default $k$-mer settings. Enabling of the "careful" option minimized errors during genome assembly followed by Quast v3.1 quality assessment of assemblies [32]. Sequence reads were preprocessed according to the following criteria; 1) minimum sequence quality Q20,2) minimum read lengths of $35 \mathrm{bp}$, and 3) removal of low quality reads from the $5^{\prime}$-end ( $20 \mathrm{bp}$ ) and $3^{\prime}$-end (5 bp). Minimum scaffold length was set as $200 \mathrm{bp}$ and scaffolds having mean assembly coverage lower than $5 x$ were discarded. The sequence coverage was set to $50 x$.

\subsubsection{Verification of species identifications.}

The bacterial identities were post-sequencing verified using the 16S rRNA gene sequence. The 16S rRNA gene sequences of clinical strains were predicted using SpeciesFinder [33] and used for nucleotide BLAST [34] against NCBI GenBank. The identifications were evaluated using BLAST percent identities, differences between maximum score of best and second best taxon matches, and minimum E-values of 0.001 .

\subsection{Pan- and core-genome characterizations}

\subsubsection{Genome annotations and identification of pan-and core-genomes.}

Pan- and core-genomes were defined using PAN-genome analysis based on FUNctional PROfiles, PanFunPro [35]. Genes were predicted and translated into amino acid sequences using Prodigal v2.5 [36]. Each protein sequence was scanned against three protein databases with InterProScan [37] in the following order; PfamA [38], TIGRFAM [39], and SUPERFAMILY [40] to identify functional protein domains. Genes translated into protein sequences with identical functional protein domains were categorized as belonging to the same protein family. Proteins without identified functional domains were clustered using CD-hit [41] according to at least $60 \%$ amino acid identities. For each genome, a collection of the annotated genes and the CD-hit clustered sequences constituted the genome profiles, and the complete collection of genome profiles from all strains represented the pan-genome. 
211 The number of predicted genes for each strain was visualized in a genome plot along with the fraction of 212 genes with protein domains of annotated function, protein domains with unknown function, and with no 213 functional protein domains identified.

214 Genes found to be present in all of the analyzed genomes were categorized as belonging to the core215 genome using PanFunPro2apply of PanFunPro [35] and visualized in a genome plot. Each collection of 216 translated core-gene sequences were clustered using CD-hit [41] to ensure homology according to at least $21760 \%$ amino acid identities and $60 \%$ coverage. Core-genes passing the clustering criteria were globally 218 aligned in MUSCLE v3.8.425 [42] and translated core-genes with less than $30 \%$ conserved amino acid sites 219 were not taken into considerations as core-genes.

\subsubsection{Pan-genomic proteome comparison.}

222 Genomic relationships of strains were analyzed using PanFunPro predicted pan-genes. These genes were 223 used for construction of a presence-absence matrix of genes within all genomes using 224 PanGenome2Abundance of PanFunPro [35]. Genomic clustering of strains were statistically analyzed using 225 Pearson correlation of the matrix. The correlation was illustrated as a heatmap where the correlation coefficient was color assigned.

\subsubsection{Proteome conservations.}

229 The level of proteome conservations within each species were analyzed by pairwise all-against-all 230 comparisons of protein domain annotations. For each comparison, the absolute number of shared protein 231 families out of the total number of protein families were shown and converted into percentages. The 232 genomic relatedness of two proteomes were demonstrated as a color assigned matrix plot, and the darker 233 coloring, the higher percent identities and the higher degree of proteome conservations.

\subsection{Phylogenetic relationships}

\subsubsection{Core-gene phylogeny.}

238 The phylogenetic relationships of the clinical $A$. urinae strains were analyzed using common core-genes 239 within all 40 clinical A. urinae genomes. The PanFunPro predicted and subsequent homology verified protein 240 sequences, encoded by the core-genes, were concatenated and multiple sequence aligned using MUSCLE 241 v.3.8.425 [42]. jModelTest v2.1.10 [43] predicted the Le \& Gascuel amino acid substitution model as the 242 best-fit substitution model for the core-tree construction. PhyML V3.1 [44] generated the maximum 
likelihood phylogenetic tree and the tree robustness was evaluated using 100 bootstrap replicates. The tree was visualized in CLC bio's Genomics Workbench v9.0 (www.qiagenbioinformatics.com).

\subsubsection{SNPs phylogeny.}

The phylogenetic relationships of the $40 \mathrm{~A}$. urinae strains were verified using single-nucleotide polymorphisms (SNPs). SNPs were determined using the CSI Phylogeny (www.cge.cbs.dtu.dk/services/CSIPhylogeny) [45] by mapping of raw sequence reads against a reference genome. Three phylogenetic trees were generated, either by using the A. urinae CCUG $36881^{\top}$ type strain (complete genome), the clinical A. urinae ACS-120V-Col10a (complete genome), or the clinical A. urinae AU3 (draft genome) as reference genomes. Calling of SNPs and validations were performed according to default settings of CSI Phylogeny.

SNPs passing the quality thresholds were concatenated to SNP sequences. Phylogenetic trees were created using the jModelTest [43] which predicted generalized time reversible nucleotide substitution model, as the most suitable substitution model for the dataset. The maximum likelihood trees in was generated using PhyML V3.1 [44]. Robustness of tree topologies were evaluated using bootstrap replicates of 100 and visualized in CLC bio's Genomics Workbench v9.0.

\subsection{Comparison of pan- and core-genes with Virulence Factors of Bacterial Pathogens}

PanFunPro predicted pan- and core-genes were translated into protein sequence and aligned against the protein dataset of Virulence Factors of Bacterial Pathogens (VFDB) [46] using BLASTP v2.2.31 [34]. The protein dataset, only composed of experimentally verified virulence factors, was downloaded May $27^{\text {th }} 2016$.

Translated pan- and core-genes with VFDB hit bitscore values higher than 90, E-values lower than 0.001 and BLASTP amino acid sequence identities higher than $30 \%$ were included in the analysis. Pan-genes with multiple VFDB hits were manually curated using at least $30 \%$ BLASTP amino acid identities between the query and subject sequence. The query sequences were the PfamA, TIGRFAM, and SUPERFAMILY annotated and CD-hit clustered translated genes. Subject sequences were VFDB virulence protein sequences. Only translated pan-gene homologs with the highest bitscore values against a translated VFDB virulence gene were taken into account. Core-genes with multiple VFDB hits were sorted using an in-house Perl script, in which only gene with the highest bitscore values were taken into account.

Grouping of $A$. sanguinicola and $A$. urinae putative virulence gene homologs were accomplished according to VFDB assigned functional keywords for an overall genomic characterization of putative virulence genes. 


\subsection{Bacterial Capsular Polysaccharide}

278 2.5.1 Search for CPS gene homologs within genomes of A. urinae ACS-120-V-Col10a and A. urinae AU3.

279 CPS associated gene homologs were searched within the public available A. urinae ACS-120-V-Col10a and A. 280 urinae AU3 genomes. These genomes were subjected to BLASTX analysis against CPS associated genes of 281 VFDB [46]. The BLASTX analysis was performed in CLC bio's Genomics Workbench v9.0 using E-values of 2820.001 , bitscore values higher than 90, and minimum amino acid sequence identities of $30 \%$. Genes with 283 multiple VFDB CPS gene mappings were sorted by only taking the BLAST hit with the highest bitscore value.

\subsubsection{Mapping of CPS gene homologs within assembled genomes for prediction of putative CPS loci.}

All the identified CPS gene homologs were plotted against the assembled $A$. sanguinicola and $A$. urinae genomes according to gene positions. Genomic regions with high abundance of CPS associated gene homologs were extracted and identified as putative CPS loci.

\subsubsection{CPS structural organization analysis.}

Mapping of gene homologs to the same VFDB CPS gene homologs were color assigned with the same color and side-by-side visualized in Geneious v9.1.6 [47].

Protein sequences of the initial four $A$. urinae gene homologs of $c p s 4 A, c a p 8 A$, cap8B, and cap8C, which constituted the common CPS loci region were subjected to four global protein sequence alignments to determine sequence identities using the MUSCLE v.9.1.6 [42].

The common CPS regions were followed by regions of variable sizes and genetic contents, hence defined as the variable CPS region. Genes positioned within the variable CPS loci regions and without VFDB assigned CPS annotations were subjected to BLASTX analysis for functional characterizations against the nonredundant protein sequence database of NCBI [34]. Only BLAST hits with E-values lower than 0.001 were taken into considerations.

\subsection{Heat shock protein 60}

The PanFunPro predicted A. sanguinicola and A. urinae Hsp60 homolog protein sequences (541-542 amino acids), encoded by the $h t p B$ gene, were compared against the Hsp60 protein sequence of the virulent $A$. viridans var. homari (184 amino acid partial sequence, AAM88526.1) to calculate sequence identities. The comparisons were made using the protein BLAST implementation in CLC bio's Genomics Workbench v9.0. 
309

\subsection{Adhesion associated gene homologs and cell wall signaling and anchoring}

The presence of signal peptides were predicted using SignalP v4.1 (www.cbs.dtu.dk/services/SignalP/) [48] and PSORTb v3 (www.psort.org/) [49]. The presence of cell wall anchoring protein domains were predicted using the TMHMM Server v2.0 (www.cbs.dtu.dk/services/TMHMM/) [50].

This study was approved by the Danish Data Protection Agency (J.nr. 2012-41-0240).

\section{RESULTS}

\subsection{Species verification by $16 \mathrm{~S}$ rRNA gene sequence analysis and features of genomic sequence data}

\subsubsection{Confirmation of species identifications.}

Forty-eight Danish clinical strains of $A$. sanguinicola $(n=8)$ and A. urinae $(n=40)$ (Supplementary A) were subjected to whole-genome analysis and genomic characterizations, including the corresponding type strains.

Identification to the species level using MALDI-TOF MS (score above 2.0) were post-sequencing verified using BLASTN sequence analysis of the $16 \mathrm{~S}$ rRNA gene sequence against NCBI GenBank.

More than $99 \%$ sequence identities were observed between the clinical A. sanguinicola 16S rRNA gene sequence and the public available type strain A. sanguinicola CCUG $43001^{\top}$ (BLAST maximum alignment score $2,835-2,841$ ), and between the clinical $A$. urinae strains and the public available type strain $A$. urinae CCUG $36881^{\top}$ (BLAST maximum alignment score 2,804-2,837). BLAST maximum alignment score value differences between the best and second best taxon matches were 316-366.

\subsubsection{Features of genomic sequence data.}

The number of de novo assembled scaffolds ranged from 17-44 and 12-58 for the clinical A. sanguinicola and A. urinae strains, respectively (Table 1). Genome sizes of $A$. sanguinicola strains were between $2.06 \mathrm{Mb}$ to $2.12 \mathrm{Mb}$ with GC-contents of 47.4-47.6\%. A. urinae genome sizes ranged from $1.93 \mathrm{Mb}$ to $2.44 \mathrm{Mb}$ with GCcontents of 41.6-42.6\%. The 1984-2004 and 2010-2015 strains had average genome sizes of 1,947,525 bp (range 1.93-2.01 Mb) and 2,032,841 bp (1.93-2.44 Mb), respectively, which corresponded to an average increase of 86,000 bp genetic material in the 2010-2015 strains.

The type strains of $A$. sanguinicola CCUG $43001^{\top}$ and $A$. urinae CCUG $36881^{\top}$ had genome sizes of $2.03 \mathrm{Mb}$ (GC-content $47.6 \%)$ and $1.97 \mathrm{Mb}$ (GC-content $42.6 \%$ ), respectively (Table 1). 
340 Genomes of all A. sanguinicola strains and the corresponding type strain consisted of 1,783-1,905 genes and $341 \quad 1,708-2,256$ genes were identified within the genomes of $A$. urinae. The genome annotations revealed a high 342 proportion of genes which encoded proteins with known annotated functional protein domains (78-84 \%), 343 with protein domains of unknown function (7-8 \%), and proteins without annotated protein domains (8-14 $344 \%)$.

345 
Table 1. Clinical and genomic characteristics of all clinical and type strains belonging to the A. sanguinicola and A.

urinae species.

\begin{tabular}{|c|c|c|c|c|c|c|}
\hline Characteristics & 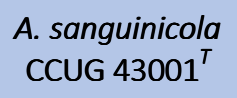 & $\begin{array}{l}\text { A. sanguinicola } \\
\text { (all strains) }\end{array}$ & $\begin{array}{c}\text { A. urinae } \\
\text { CCUG } 36881^{T}\end{array}$ & $\begin{array}{l}\text { A. urinae } \\
\text { (all strains) }\end{array}$ & $\begin{array}{l}\text { A. urinae } \\
1984-2004\end{array}$ & $\begin{array}{c}\text { A. urinae } \\
2010-2015\end{array}$ \\
\hline \multicolumn{7}{|l|}{ Clinical feature } \\
\hline Strain category & Type strain & Clinical strains & Type strain & Clinical strains & \multicolumn{2}{|c|}{ Clinical strains } \\
\hline Country of isolation & Denmark & Denmark & Denmark & Denmark & \multicolumn{2}{|c|}{ Denmark } \\
\hline Year of isolation & $1999^{1}$ & 2009 to 2015 & $1984^{2}$ & 1984 to 2015 & 1984 to 2004 & 2010 to 2015 \\
\hline Strains (patients) & 1 & $8(5)$ & 1 & $40(32)$ & $20(18)$ & $20(14)$ \\
\hline $\begin{array}{l}\text { Patient mean age yrs. } \\
\text { (range) }\end{array}$ & - & $75(62-87)$ & - & $73(10-94)$ & $74.8(56-85)$ & $70.7(10-94)$ \\
\hline $\begin{array}{l}\text { Gender ratio } \\
\text { Male:Female:Unknown }\end{array}$ & - & 2:3:0 & - & $18: 8: 6$ & $8: 4: 6$ & 10:4:0 \\
\hline Source of isolation & Blood & Urine and blood & Human urine & $\begin{array}{l}\text { Urine, blood and } \\
\text { heart valve }\end{array}$ & $\begin{array}{l}\text { Urine, blood and } \\
\text { heart valve }\end{array}$ & $\begin{array}{c}\text { Urine and } \\
\text { blood }\end{array}$ \\
\hline Type of infection & Sepsis & UTI and bacteremia & UTI & $\begin{array}{l}\text { UTI, bacteremia, } \\
\text { and IE }\end{array}$ & $\begin{array}{l}\text { UTI, bacteremia, } \\
\text { and IE }\end{array}$ & $\begin{array}{l}\text { UTI and } \\
\text { bacteremia }\end{array}$ \\
\hline \multicolumn{7}{|l|}{ Genomic feature } \\
\hline Genome size (Mb) & 2.03 & $2.06-2.12$ & 1.97 & $1.93-2.44$ & $1.93-2.01$ & $1.93-2.44$ \\
\hline $\begin{array}{l}\text { Average genome size } \\
\text { (bp) }\end{array}$ & - & - & & - & $1,947,525$ & $2,032,841$ \\
\hline Scaffolds & 1 & $17-44$ & 1 & $12-58$ & $26-40$ & $12-58$ \\
\hline GC-content (\%) & 47.6 & $47.4-47.6$ & 42.6 & $41.6-42.6$ & $42.4-42.6$ & $41.6-42.5$ \\
\hline Genes & $1,783^{3} / 1,838^{4}$ & $1,783-1,905^{3}$ & $1,739^{3} / 1,801^{4}$ & $1,708-2,256^{3}$ & $1,725-1,800^{3}$ & $1,708-2,256^{3}$ \\
\hline $\begin{array}{l}\text { Core-genes (amino } \\
\text { acid percent identity) }\end{array}$ & - & 1,170 & - & 907 & $\begin{array}{c}1,191 \\
(99.4-100 \%) \\
\end{array}$ & $\begin{array}{c}1,011 \\
(96.6-100 \%) \\
\end{array}$ \\
\hline $\begin{array}{l}\text { Unique intra-period } \\
\text { core-genes }\end{array}$ & - & & - & - & 204 & 24 \\
\hline $\begin{array}{l}\text { Common core-genes } \\
\text { (amino acid length) }\end{array}$ & - & & - & - & $987(312,235$ & nino acids) \\
\hline
\end{tabular}

UTI, Urinary tract infection.

$\mathrm{IE}$, Infective endocarditis.

${ }^{1}$ Isolated in 1999 and characterized in 2001.

${ }^{2}$ Isolated in 1984, characterized in 1989, and reclassified in 1992.

${ }^{3}$ Number of genes according to genome annotation using the PanFunPro pipeline [35].

${ }^{4}$ Number of genes according to genome annotation using the NCBI Prokaryotic Genome Annotation Pipeline [51].

\subsection{Pan- and core-genome characterizations, proteome conservations, and phylogeny}

\subsubsection{Pan-genome analysis.}

The total number of genes for all strains of $A$. sanguinicola were 16,678 genes and for strains of $A$. urinae 72,930 genes, including the type strains in both cases. The total number of genes for both species was 89,608 genes, of which 2,360 unique pan-genes. These genes were used to analyze the genomic relatedness of all strains with a presence-absence analysis of the pan-genes across all strains (Figure 1). 
362 Overall, high intra-species clustering was observed within both species and low clustering was observed 363 between both species (correlation coefficient below 0.4). The intra-species clustering was highest within 364 strains of $A$. sanguinicola (green, correlation coefficient 0.9-1) and within 1984-2004 isolated A. urinae 365 strains (pink, correlation coefficient 0.9-1). The 2010-2015 isolated $A$. urinae strains (blue) showed internal 366 heterogeneity (correlation coefficient 0.6-1). All the paired strains showed very high genomic clustering 367 (correlation coefficient 0.9-1).

368
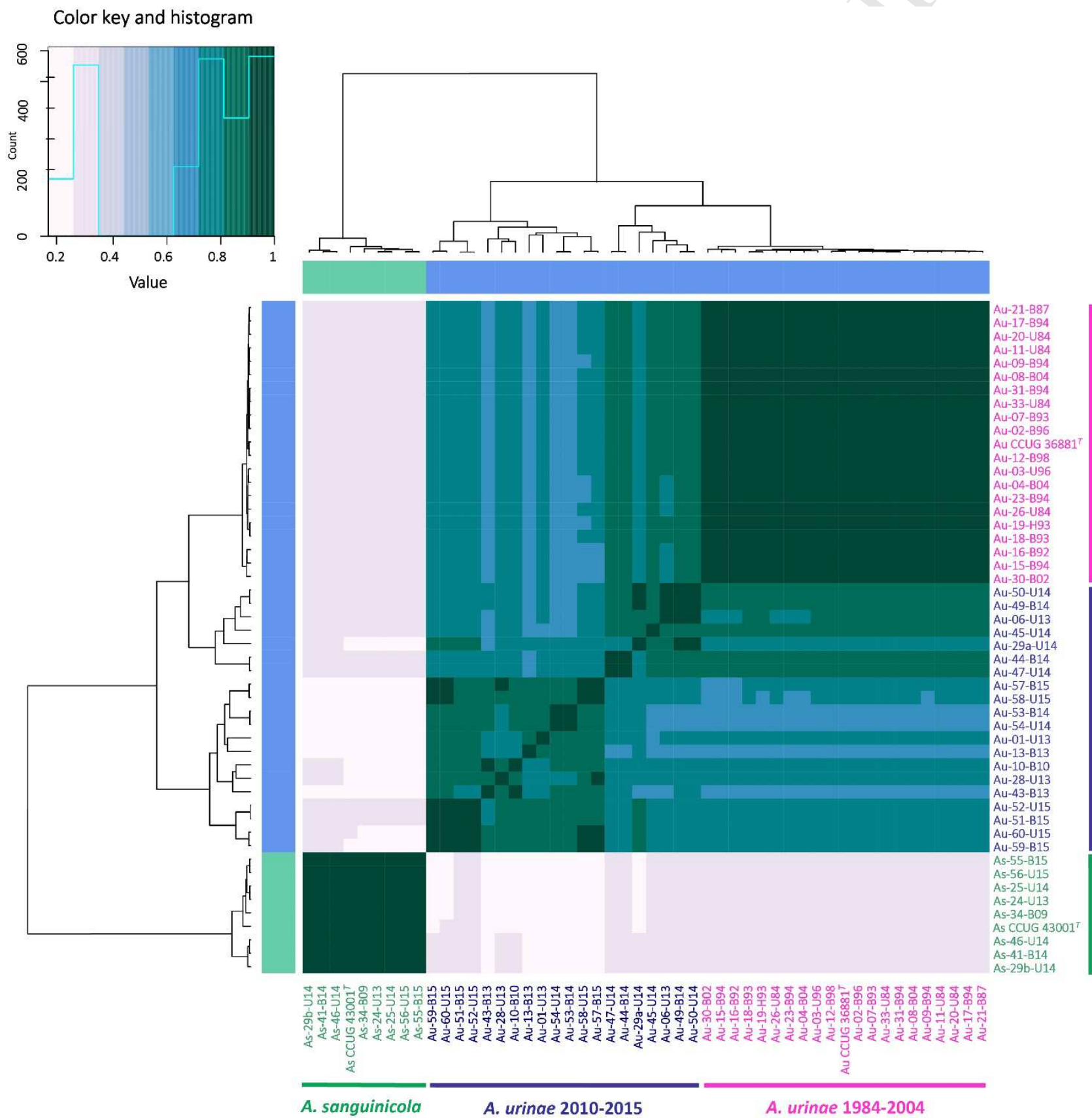
Figure 1. Clustering of $A$. sanguinicola and $A$. urinae strains using Pearson correlation of the presence-absence matrix of the 2,360 unique pan-genes within both species. The highest correlation and genomic clustering was observed at correlation coefficient 1 (darkest coloring) and lowest at 0 (brightest coloring). Strains of $A$. sanguinicola showed high genomic clustering (green, correlation coefficient 0.9-1) and internal heterogeneity within A. urinae strains (blue and pink, correlation coefficient 0.6-1). The A. urinae 1984-2004 showed high genomic clustering (pink, correlation coefficient 0.9-1) and heterogeneity within the $A$. urinae 2010-2015 strains (blue, correlation coefficient 0.6-1). Low clustering was observed between the two species (correlation coefficient below 0.4). All the paired strains showed very high genomic clustering (correlation coefficient 0.9-1).

\subsubsection{Core-genome analysis.}

Highly conserved core-genomes were observed within both species as the number of core-genes decreased slightly as more genomes were added. The core-genomes reached a plateau stage through both species.

The number of PanFunPro predicted core-genes for clinical and the type strain of $A$. sanguinicola started from 1,359 core-genes and dropped to 1,260 core-genes when genomes of all $A$. sanguinicola strains were included. Core-gene homology was further verified using $60 \%$ protein sequence identity across $60 \%$ sequence coverage and more than $30 \%$ sequence identities, which reduced the core-gene number to 1,170 genes for $A$. sanguinicola strains (Table 1 ). In case of the clinical and the $A$. urinae type strain, the number of core-genes started from 1,314 genes and dropped to 1,023 genes when genomes of all $A$. urinae strains were included. Using the same homology verification criteria as in case of $A$. sanguinicola core-genes, the number was reduced to 907 core-genes (Table 1 ). Without the $A$. urinae type strain, the remaining 40 clinical A. urinae strains shared 987 core-genes (312,235 amino acids with overall 95.7-100 \% amino acid identities). In case of the 1984-2004 and 2010-2015 A. urinae strains, the number of core-genes were determined as 1,191 core-genes (99.4-100 \% amino acid identity) and 1,011 core-genes (96.6-100 \% amino acid identity), respectively. A total number of 204 core-genes were unique for only the 1984-2004 strains and 24 coregenes for the 2010-2015 strains.

The number of common core-genes, which fulfilled the homology verification criteria using $60 \%$ sequence identities, were 81 genes for all A. sanguinicola and A. urinae strains.

\subsubsection{A. urinae proteome conservations of $1984-2004$ and 2010-2015 A. urinae strains.}

Between 1,725-1,800 and 1,708-2,256 genes were predicted within the 1984-2004 and the 2010-2015 strains, respectively (Table 1). These genes were evaluated and classified into 1,208 and 1,347 protein families for both species, respectively. Intra-period comparison of protein families showed high degree of proteome conservations as 96.4 to $99.7 \%$ protein families were shared within the 1984-2004 strains (Supplementary material B). Higher proteome variations were observed within the 2010-2015 strains as 
404

405

406

407

408

409

410

411

412

413

414

415

416

417

418

419

420

421

422

423

424

425

426

427

428

429

74.3-99.8 \% of the protein families were shared. Inter-period comparison of the 1984-2004 and 2010-2015 strains showed 74.7-87.8 \% identities of shared protein families. Each of the paired strains exhibited 99.2$99.8 \%$ identities.

3.2.4. A. urinae phylogeny based on common core-genes and SNPS.

The 987 common core-genes within all 40 clinical $A$. urinae strains were used to demonstrate the phylogenetic relatedness (Figure 2). These 987 core-genes corresponded to 312,234 amino acids and with 95.7-100\% sequence identities. Strains were color assigned according to type of infection: UTIs (yellow), bacteremia (red), and IE (blue). For the 1984-2004 and 2010-2015 strains, these 987 core-genes showed 99.4-100\% and 96.6-100\% amino acid sequence identities, respectively.

The phylogenetic analysis showed no clustering related to the disease entity (UTIS, bacteremia, and IE). Two major clustering were observed, one consisting of the 1984-2004 strains and the second cluster consisted of the 2010-2015 strains, of which the main branch separating these two groups of strains was supported by bootstrap values of 100. Sub-clusterings were shown within the 2010-2015 cluster and also supported by bootstrap values of 100. Each of the eight paired A. urinae strains (marked with colored dots), from blood and urine samples from seven patients and from one blood and heart valve sample from one patient, clustered very close to each other and supported by bootstrap values of 100 .

Identical clustering patterns of the 1984-2004 and 2010-2015 A. urinae strains were observed when SNPs were used to generate the phylogenetic relationships, showing two major clusters (Supplementary material C, Figure A, B, and C). Each of the paired A. urinae isolates were likewise clustered very close to each other.

When using the A. urinae CCUG $36881^{\top}$ genome (isolated in 1984) as a reference genome for SNP callings, 20,694 SNPs were predicted and this reference strain clustered within the 1984-2004 cluster with strains from the same time period of isolation (Supplementary materials C, Figure A). A. urinae ACS-120-V-Col10a (isolated in 2007) and A. urinae AU3 (isolated in 2010) showed 22,608 SNPs and 21,302 SNPs, respectively, and clustered within the 2010-2015 cluster (Supplementary materials C, Figure B and C). 


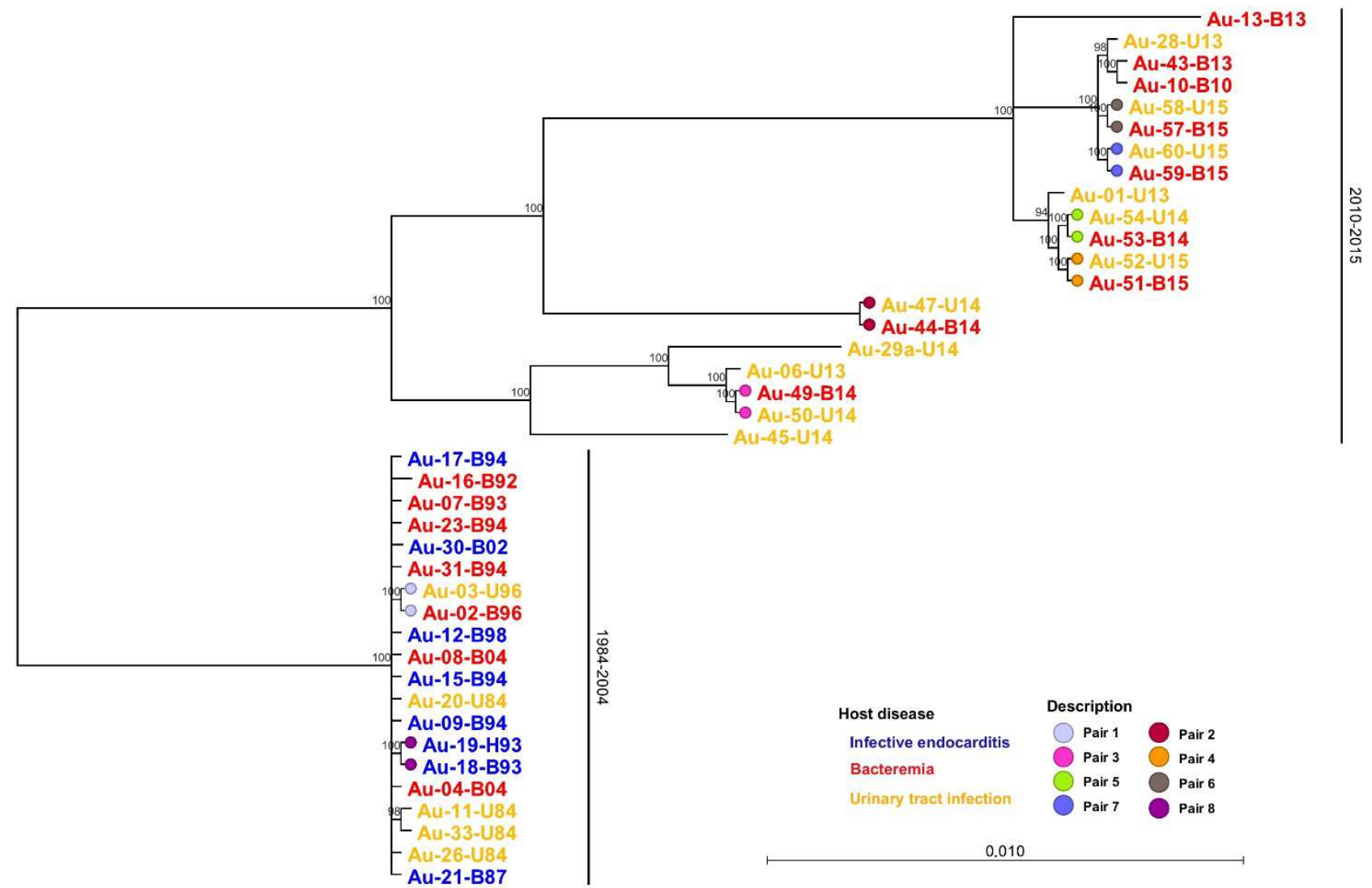

430

431

432

433

434

435

436

437

438

439

440

441

442

443

444

Figure 2. Core-genome phylogeny of the 40 clinical $A$. urinae strains based on the 987 translated common coregenes (corresponding to 312,235 amino acids). The tree showed two major clustering of strains, one with the 1984-2004 strains and the other with strains from 2010-2015. Sub-clustering was observed within the 2010-2015 cluster. Strains were color assigned according to type of infections of UTIs (yellow), bacteremia (red), and IE (blue). The last three characters of each strain identifier represented the source of strain isolation, blood (B), urine $(U)$ or heart valve $(H)$ followed by the year of strain isolations. Branching of the maximum likelihood tree was supported by bootstrap replicates of 100 and only bootstrap values higher than 90 were shown. Branch lengths were given as substitutions per site. Clustering of the eight paired strains (marked with colored dots and isolated from blood and urine samples of seven patients and blood and heart valve sample of one patient) were very close to each other and supported by bootstrap values of 100 .

\subsection{Comparison of pan- and core-genes with Virulence Factors of Bacterial Pathogens}

\subsubsection{Virulence gene homologs from the pan-and core-genomes.}

The 16,678 pan-genes of $A$. sanguinicola and 72,930 pan-genes of $A$. urinae contained 12 and 20 VFDB homolog virulence genes, respectively. Thirty-four out of 1,170 A. sanguinicola core-genes were identified as VFDB homologs and similarly 24 genes out of 907 A. urinae core-genes. Only one common core-gene, which 
448 encodes a HtpB protein (around 53-56 \% protein sequence identities, Table 2), was predicted as a putative 449 virulence gene of the 81 common core-genes of $A$. urinae and $A$. sanguinicola using at least $60 \%$ protein 450 sequence identities.

451 VFDB assigned keywords for functional characterization were used for an overall distribution of $A$. 452 sanguinicola and A. urinae specific pan- and core-genes (Supplementary material D). The highest number of 453 genes within one category was observed for genes associated with antiphagocytosis (15 genes in $A$. 454 sanguinicola and between 11-16 genes in A. urinae strains). This was followed by genes associated with 455 adherence (four genes in $A$. sanguinicola and five genes in $A$. urinae) and endotoxins (six genes in $A$. 456 sanguinicola and five genes in A. urinae). Genes were also associated with intracellular growth/survival 457 (three genes in A. sanguinicola and two genes in A. urinae) and stress proteins (four genes in A. sanguinicola 458 and three genes in A. urinae). According to VFDB keywords, only strains of A. sanguinicola encoded gene 459 homologs associated with biofilm formation (one gene) and beta-hemolysin/cytolysin (three genes). The 460 miscellaneous group included genes related to iron and magnesium uptake/acquisition, surface protein 461 anchoring, secretion system, regulation, and genes with uncharacterized function according to VFDB 462 keyword designations (10 genes in A. sanguinicola and eight genes in A. urinae).

463 Antiphagocytosis, adherence, and biofilm formation associated proteins are known important virulence 464 factors during bacterial infections. Translated pan- and core-gene homologs associated with these three 465 virulence properties were selected for further characterizations. Each VFDB homolog pan- and core-gene is 466 represented with protein sequence identities against the respective VFDB hit along with VFDB annotations 467 and keyword designations (Table 2). 
Table 2. A. sanguinicola and A. urinae virulence gene homologs of pan- and core-genes (protein level), involved in antiphagocytosis, adherence, and biofilm formation.

\begin{tabular}{|c|c|c|c|c|}
\hline \multirow{2}{*}{ Reference strain } & \multirow{2}{*}{ VFDB annotation } & \multirow{2}{*}{$\begin{array}{l}\text { VFDB } \\
\text { gene }\end{array}$} & A. sanguinicola ${ }^{1}$ & A. urinae ${ }^{2}$ \\
\hline & & & Sequence identity in \% ( $n)$ & Sequence identity in \% (n) \\
\hline \multicolumn{5}{|c|}{ VFDB category: Antiphagocytosis } \\
\hline \multirow{7}{*}{$\begin{array}{l}\text { S. aureus ssp. } \\
\text { aureus MW2 }\end{array}$} & CPS protein Cap8A & cap8A & $34.3(9)$ & $30.4-32.0(41)$ \\
\hline & CPS protein Cap8B & cap8B & $36.0-36.2(9)$ & 37.9-39.2 (41) \\
\hline & CPS protein Cap8C & $\operatorname{cap} 8 \mathrm{C}$ & - & $43.6-45.6(41)^{3 a}$ \\
\hline & CPS protein Cap8D & $\operatorname{cap} 8 D$ & $48.0-48.3(9)$ & $46.9-47.4(24) \& 63.7(3)^{4 a}$ \\
\hline & CPS protein Cap8F & cap8F & $54.7(9)$ & $53.7-53.9(22)$ \\
\hline & CPS protein Cap8G & cap8G & $50.8(9)$ & 50.8-51.9 (22) \\
\hline & CPS protein Cap8N & cap8N & $38.4(9)$ & $38.9-40.7(27)$ \\
\hline \multirow{6}{*}{$\begin{array}{l}\text { S. pneumoniae } \\
\text { TIGR4 }\end{array}$} & CPS protein Cps4A & $\operatorname{cps} 4 A$ & - & $35.3-36.1(40) \& 33.3-42.9(1)^{4 b}$ \\
\hline & CPS protein CPs4E & $\operatorname{cps} 4 E$ & $60.4(9)$ & $57.8-59.4(23) \& 57.3(4)^{36}$ \\
\hline & CPS protein Cps4F & $\operatorname{cps} 4 F$ & $33.9-34.2(9)$ & $33.2-33.4(22)$ \\
\hline & CPS protein $\mathrm{Cps} 4 \mathrm{H}$ & $\operatorname{cps} 4 \mathrm{H}$ & -4 & $30.6-31.4(5)$ \\
\hline & CPS protein Cps4l & cps4l & - & $63.0(2)$ \\
\hline & CPS protein Cps4J & cps4J & $70.6-70.9(9)$ & $70.6(21) \& 74.4(1)^{4 c}$ \\
\hline \multirow{3}{*}{ E. faecalis V583 } & Undecaprenyl diphosphate synthase & cpsA & $49.8(9)$ & $51.4(41)$ \\
\hline & Phosphatidate cytidylyltransferase & $\operatorname{cps} B$ & $41.7(9)$ & $42.2-42.9(41)$ \\
\hline & UDP-galactopyranose mutase & cpsl & 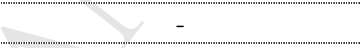 & $60.5(14)$ \\
\hline \multirow{5}{*}{$\begin{array}{l}\text { S. agalactiae } \\
2603 \mathrm{~V} / \mathrm{R}\end{array}$} & Glycosyl transferase CpsE & cpsE & 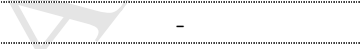 & $33.9(12) \& 58.5-71.4(2)^{4 d}$ \\
\hline & Glycosyl transferase CpsJ & cps」 & $34.9-35.3(9)$ & - \\
\hline & CPS protein CpsL & cpsL & - & $32.7(14)$ \\
\hline & Glycosyl transferase CpsO & cpsO & $45.7(9)$ & - \\
\hline & $\mathrm{N}$-acetyl neuramic acid synthetase NeuB & neuB & - & $39.8-40.4(41)$ \\
\hline \multirow{2}{*}{ S. pyogenes M1 } & UDP-glucose 6-dehydrogenase HasB & hasB & - & $52.3(24)$ \\
\hline & UDP-glucose pyrophosphorylase HasC & hasC & $66.2-66.6(9)$ & $50.7-51.9(41)$ \\
\hline $\begin{array}{l}\text { C. jejuni ssp. jejuni } \\
\text { NCTC } 11168\end{array}$ & UDP-glucose 6-dehydrogenase KfiD & $k f i D$ & $49.6-49.8(9)$ & - \\
\hline \multicolumn{5}{|c|}{ VFDB category: Adherence } \\
\hline $\begin{array}{l}\text { L. pneumophila } \\
\text { ssp. pneumophila } \\
\text { str. Philadelphia } 1\end{array}$ & Hsp60, 60K heat shock protein HtpB & $h t p B$ & $56.1-56.3(9)$ & $53.6-54.0(41)$ \\
\hline \multirow{2}{*}{$\begin{array}{l}\text { L. monocytogenes } \\
\text { EGD-e }\end{array}$} & Fibronectin-binding protein FbpA & $f b p A$ & $41.5-41.8(9)$ & - \\
\hline & Listeria adhesion protein LAP & lap & - & $54.4-54.7(41)$ \\
\hline $\begin{array}{l}\text { S. agalactiae } \\
2603 \mathrm{~V} / \mathrm{R}\end{array}$ & Laminin-binding surface protein Lmb & $I m b$ & $32.4(9)$ & $56.2-56.9(41)$ \\
\hline S. pyogenes M1 & Fibronectin-binding protein Fbp54 & fbp54 & - & $42.2-43.1(41)$ \\
\hline V. vulnificus YJ016 & Immunogenic lipoprotein A IIpA & $i / p A$ & $38.0(9)$ & $38.1-39.2(41)$ \\
\hline \multicolumn{5}{|c|}{ VFDB category: Biofilm formation } \\
\hline E. faecalis V583 & Sugar-binding transcriptional regulator & bopD & $31.8-32.2(9)$ & - \\
\hline
\end{tabular}
CPS, Capsular polysaccharide.

$472{ }^{1}$ A. sanguinicola strains: Eight clinical and one type strain.

$473 \quad{ }^{2}$ A. urinae strains: Forty clinical and one type strain.

$474{ }^{3 a / b}$ Gene homologs of a) cap8C (Au-18-B93 and Au-19-H93) and b) cps4E (Au-02-B96, Au-03-U96, Au-12-B98, and 475 Au-15-B94) were predicted as shorter genes compared to the remaining cap8C and cps4E homolog genes of $A$. 476 urinae strains, respectively. 
4a/b/c/d Gene homologs of a) cap8D (Au-06-U13, Au-49-B14, and Au-50-U14), b) cps4A (Au-06-U13), c) cps4J (Au45-U14), and d) CPSE (Au-43-B13 and Au-10-B10) were predicted as two partial and shorter genes instead of one full length gene compared to the remaining $A$. urinae genes of the particular gene homolog.

\subsubsection{Bacterial capsular polysaccharide gene homologs involved in evasion of immune phagocytosis.}

The CPS gene homologs as identified in A. sanguinicola and A. urinae strains were described in six bacterial species; Staphylococcus aureus, Streptococcus pneumoniae, Enterococcus faecalis, S. agalactiae, S. pyogenes, and Campylobacter jejuni (Table 2). A. sanguinicola strains consisted of 15 CPS gene homologs and between 11-16 CPS gene homologs were identified in A. urinae strains. The public available A. urinae ACS-120-VCol10a and A. urinae AU3 consisted of 13 and 16 CPS gene homologs, respectively. The majority of the CPS gene homologs were described in S. aureus ssp. aureus MW2 (cap8 genes) and S. pneumoniae TIGR4 (cps4 genes). The highest percent identity was observed for the S. pneumoniae TIGR4 cps4J gene homolog with 70.6-70.9 \% for A. sanguinicola and 70.6-74.4 \% for A. urinae strains.

Mapping of CPS gene homologs within the assembled genomes demonstrated regions with high abundance of CPS gene homologs in all the strains, whereof identified as putative CPS loci (Figure 3). These genes were positioned in the same orientation of translation and ordered behind each other with short distances to neighboring genes. Four CPS gene homologs of A. sanguinicola strains ( $\operatorname{cps} A, \operatorname{cps} B$, hasC, and $k f i D)$ and four of $A$. urinae strains ( $c p s A, c p s B$, neuB, and has $C$ ) were located outside of the putatively predicted CPS loci regions and presumable not involving in CPS.

The CPS loci sizes were estimated between 12,800 to 19,500 bp, from positioning of CPS gene homologs until flanking by non-CPS associated genes. The number of genes within the CPS loci varied from 13 to 19 genes, of which 7-12 genes were identified as CPS gene homologs. The genetic CPS loci arrangements showed one type of CPS loci for $A$. sanguinicola and five different types for $A$. urinae strains, the latter allocated into two major and three minor groups (Figure 3). Major group I was composed of all $A$. urinae strains from 1984-2004 and the A. urinae CCUG 36881 ${ }^{\top}$ and major group II of 14 of the 20 strains from 20102015. The three minor groups were composed of one 2014 isolate (minor group I), two 2014 isolates (minor group II), and one 2013 and two 2014 isolates (minor group III). The A. urinae ACS-120-V-Col10a constituted a different CPS locus type and due to contig truncation the CPS locus of $A$. urinae AU3 was only partially identified.

Analysis of the CPS loci throughout all A. sanguinicola strains showed the initial two CPS gene homologs, cap8A (100\% protein sequence identity) and cap8B (99.9-100\%) to hold annotation of transcriptionally regulatory function. The remaining CPS gene homologs within the putative CPS loci showed higher than 97.9 $\%$ protein sequence identities within all $A$. sanguinicola strains. In case of $A$. urinae strains, the initial four CPS 
511

512

513

514

515

516

517

518

519

gene homolog were identified as transcriptionally regulator proteins in all strains and identified as the common CPS region, cps4A (88.8-100 \% protein sequence identity), cap8A (92.9-100 \%), cap8B (94.9-100 \%), and cap8C (86.3-100\%). Higher protein identities were observed when the four common region CPS gene were compared within strains of major group I and within major group II (Table 3).

Table 3. Sequence identities of the four translated CPS gene homologs constituting the common CPS region of all A. urinae strains.

\begin{tabular}{|l|c|c|c|c|}
\hline \multirow{2}{*}{ CPS loci } & \multicolumn{3}{c|}{ CPS loci common region } \\
\cline { 2 - 5 } & cps4A & cap8A & cap8B & cap8C \\
\hline All A. urinae strains & $88.8-100 \%$ & $92.9-100 \%$ & $94.9-100 \%$ & $86.3-100 \%$ \\
\hline Major group I - A. urinae strains from 1984-2004 $(n=20)$ & $99.7-100 \%$ & $100 \%$ & $99.1-100 \%$ & $100 \%$ \\
\hline Major group II - A. urinae strains from 2010-2015 $(n=14)$ & $100 \%$ & $100 \%$ & $99.6-100 \%$ & $99.6-100 \%$ \\
\hline
\end{tabular}

The common CPS loci region of $A$. urinae strains were followed by a variable region with variations in size, number of genes and genetic arrangements. This region was consisting of CPS gene homologs and genes not matching any of the CPS genes of the VFDB database. The latter genes were classified into three categories by evaluation of the genome annotations and further characterizations using BLASTX against the NCBI protein database. The three categories were consisting of I) CPS associated glycosyl transferases and hypothetical glycosyl transferases; II) cell surface polysaccharide biosynthesis and CPS synthesis related proteins; and III) hypothetical proteins and proteins with unknown function. The cell surface polysaccharide biosynthesis and CPS synthesis related proteins were among others epimerases and dehydrogenases. Similarly, the A. sanguinicola CPS loci gene homologs were annotated as cell surface polysaccharide biosynthesis and CPS synthesis related proteins, glycosyl transferases, epimerases, and dehydrogenases.

The hasB gene homolog (UDP-glucose dehydrogenase) was positioned as the terminal CPS locus gene for all 1984-2004 strains (major group I), three 2014 strains (minor group I-II), the A. urinae CCUG $36881^{\top}$, and the A. urinae ACS-120-V-Col10a strains. Search for the hasB gene homolog within genomes of major group II and minor group III strains showed no hasB gene homologs. A hasB gene homolog was also identified in the $A$. urinae AU3 genome, although not positioned within the same CPS locus encoding contig. 
(n)

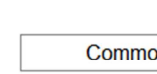

$$
12,000
$$

4,000

6,000
Variable region

Major group I 1984-2004 strains $(n=20)$ and A. urinae CCUG 36881

\section{cps4A сар8A сар8B сар8C}

cap8D

10,000

12,000

14,000

16,000

18,000

$$
\begin{array}{|c|}
\hline \text { Minor group II } \\
\hline \begin{array}{c}
\text { Au-44-B14 and } \\
\text { Au-47-U14 }(n=2)
\end{array}
\end{array}
$$

cps4A cap8A сар8B сар8C cap8D cps4E cap8N

cps4H

cps 41 Au-06-U13, Au-49-B14 and Au-50-U14 $(n=3)$

\footnotetext{
Major group II 2010-2015 strains $(n=14)$
}

\section{cps4A* cap8A сap8B cap8C cap8D cPs4E cap8N}

$\operatorname{cps} 4 \mathrm{H}$

cps4A сар8A сар8B сар8C cpsE**

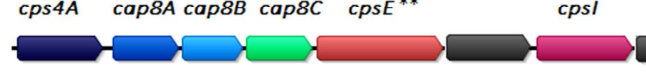

cpsL

A. urinae
A. urinae AU3


536 Figure 3. Genomic organization of CPS loci of clinical and type strains of $A$. sanguinicola and $A$. urinae, including 537 the public available A. urinae CCUG $36881^{\top}$, A. urinae ACS-120-V-Col10a, and A. urinae AU3 strains. All A. 538 sanguinicola strains were constituted of the same genomic organization of the putative predicted CPS loci. The 40 539 A. urinae strains and A. urinae CCUG $36881^{\top}$ constituted five different CPS loci, grouped into two major and three 540 minor groups. * The Au-06-U13 cps4A gene homolog was predicted as two partial and shorter genes compared to 541 the remaining cps4A gene homolog. ** The Au-10-B10 and Au-43-B13 cpsE gene homologs were predicted as two 542 partial and shorter genes compared to the remaining cpsE gene homologs.

544 3.3.3. Bacterial gene homologs involved in adhesion to host cells and biofilm formation.

545 Six gene homologs related to bacterial adherence were identified in A. sanguinicola and A. urinae genomes 546 (Table 2). Among these, four gene homologs were present in A. sanguinicola genomes and encoded the 547 immunogenic lipoprotein A (IIpA), laminin-binding surface protein (Lmb), fibronectin-binding protein (FbpA), 548 and the $60 \mathrm{~K}$ heat shock protein ( $\mathrm{HtpB})$. The A. urinae strains were containing five gene homologs which 549 encoded the fibronectin-binding protein (Fbp54), Listeria adhesion protein (LAP), and IIpA, Lmb, and HtpB as 550 with $A$. sanguinicola strains. VFDB categorized $h t p B$ of Legionella pneumophila as a bacterial adhesion 551 protein.

552 A signal peptide was only identified in $\mathrm{IpA}$ and $\mathrm{Lmb}$ proteins of $A$. sanguinicola and $A$. urinae strains, and no 553 LPXTG motif containing anchoring domains were predicted in any of the identified adhesion protein 554 homologs.

555 Comparison of Hsp60 from the virulent A. viridans var. homari strain and the $\mathrm{HtpB}$ protein of $A$. sanguinicola 556 and $A$. urinae strains showed between $79.4-82.0 \%$ protein sequence identities.

557 According to VFDB, only A. sanguinicola strains contained a biofilm-associated transcriptional regulator bopD 558 gene homolog. 
560

561

562

563

564

565

566

567

568

569

570

571

572

573

574

575

576

577

578

579

580

581

582

583

584

585

586

587

588

589

590

591

592

593

\section{DISCUSSION}

In the present study, WGS of eight $A$. sanguinicola and $40 \mathrm{~A}$. urinae strains were analyzed to characterize these genomes and to identify the potential virulence genes that cause bacterial pathogenicity.

\subsection{Genomic analysis.}

The varying number of pan- and core-genes are highly affected by the number of strains included, the degree of bacterial heterogeneity and the predefined cut-off thresholds for defining core-genes [52] as also illustrated for the strains from the two Aerococcus species examined in this study. The genetic pool of genes were lower for $A$. sanguinicola strains (16,678 genes) than for the $A$. urinae strains (72,930 genes), whereas the number of core-genes were higher for the $A$. sanguinicola strains ( 1,170 core-genes) than for strains of A. urinae strains (907 core-genes). All A. sanguinicola strains showed very close relationships taken into account of only being represented by one type strain and eight clinical strains from five patients. Marked differences were observed within all $A$. urinae strains, with respect to the average genome sizes, genomic clustering, number and sequence identity of core-genes, proteome conservations, phylogenetic analysis, and CPS loci sequences. The 20 A. urinae 1984-2004 strains, from 18 patients, were highly homogeneous compared to the 20 A. urinae 2010-2015 strains from 14 patients.

Evolution of bacteria is highly affected through genetic alternations during evolutionary processes which shapes the bacterial genomes. Homologous recombination, lateral gene transfer, as well as indel and SNP mutations are genetic events responsible for genomic diversity and shaping of bacterial populations $[53,54]$. These events can give rise to selective advantages in a bacterial species such as increased bacterial pathogenicity and adaptation for a host environment under selection pressure. In our study, analysis of unique core-genes and the subsequent core-genome phylogeny showed high genomic conservations within the 1984-2004 A. urinae strains compared to 2010-2015 strains with internal diversity. These findings were interesting in the way that these strains were belonging to the same bacterial species and only being separated by a period of six years in the strain collections. In $A$. urinae, a selective pressure, that might have taken place after 2004, could potentially explain the presence of multiple sub-clusters within the short-time span isolated 2010-2015 strains (5 years) compared to the 1984-2004 strains (20 years). Both the hostpathogen interaction, selective pressure through the use of antibiotics, and competition between microbial pathogens are factors that adds to the selectivity of beneficial genetic variations within a population [55]. Acquisition of genetic material could support an average gain of 86,000 bp in genomes of the 2010-2015 strains compared to the 1984-2004 strains, potentially increasing the genetic and proteomic variation as shown in the study.

In comparison, high level of recombination and positive selection was observed within streptococcal coregenomes. Low degree of recombination was observed in $S$. agalactiae core-genomes compared to $S$. 
594

595

596

597

598

599

600

601

602

603

604

605

606

607

608

609

610

611

612

613

614

615

616

617

618

619

620

621

622

623

624

625

626

pyogenes with high degree of core-genome recombination [56]. In S. aureus, low level of recombination was observed in the core-genomes even though being a highly pathogenic species [57]. Variations within the genomes could be dispersed across the entire genome or concentrated within specific core-genes with a selective advantages. In case of $S$. aureus genomes, recombination was often taking part in genes related to bacterial pathogenicity [57]. This kind of findings could suggest a bacterial fitness for survival and host adaptation, as suggested for Clostridium perfringens strains in an evolutionary lineage study [58].

Another aspect was if the genetic variability only were seen in Danish A. urinae isolates (local environmental pressure) of which we performed the SNPs based phylogenetic analysis. These showed the two foreign $A$. urinae isolates, one from Belgium in 2007 and one from Sweden in 2010, clustering with the Danish 20102015 isolated $A$. urinae strains. These findings may suggest that the genetic changes observed, within the recently isolated Danish $A$. urinae genomes, might be a result of a general evolutionary event. Similarly, a study from de Been et al. showed phylogenetic clustering of modern Enterococcus faecium with modern clinical isolates, by analyzing adaptive recombination events in terms of SNPs within core-genomes [59]. Marvig et al. demonstrated within-host bacterial adaptation to changing host environments and accumulation of SNPs in favor for bacterial survival and fitness of Pseudomonas aeruginosa in patients with cystic fibrosis [60]. In the latter study, SNPs were localized within the regulatory part of the bacterial genomes and in pathoadaptive genes among others CPS genes, demonstrating how positive selection for mutations might have aimed in bacterial adaptation to its host [60].

A large number of UTI causing bacteria is often associated with urosepsis, in which the pathogenic strains gets access into the bloodstream. A mortality rate of $33 \%$ was observed in hospitalized patients with cases of uncomplicated UTIs causing pathogenic Escherichia coli, leading to bacteremia [61]. The transition of a superficial site of infection to a deep site of infection is important in regards to which bacterial virulence mechanisms the UTI pathogens are taking advantages of. McNally et al. analyzed the genomic diversity of blood and urine isolates of $E$. coli from five patients with urosepsis, like we did in the current study with the eight paired $A$. urinae isolates. In four of the paired set of $E$. coli strains, the urine and blood isolates had the same sequence type, no variations were observed between each set of isolates, and only a minimal set of virulence genes were needed to establish bacteremia [62]. In the fifth E. coli urosepsis patient, two different E. coli sequence types were identified in the same urine sample and a third serotype was causing bacteremia. Based on results from McNally et al., we were not expecting to observe genomic differences within each set of the paired $A$. urinae strains and results from the current study showed highly similar set of A. urinae isolates. This indicates that superficial site of infection causing $A$. urinae isolates (from urine) were the same isolate causing a deep site of infection within the bloodstream. 


\subsection{VFDB predicted putative virulence genes.}

The current study attempted to characterize the clinical strains for the presence of virulence associated genes by comparison against a database collection of virulence factors, VFDB [46]. In this way, we only expected to identify already known virulence genes and factors as the VFDB database was consisting of. Until now, no UTI or IE associated virulence genes were characterized within genomes of $A$. sanguinicola and $A$. urinae strains.

\subsubsection{Bacterial capsular polysaccharide genes.}

Within genus Aerococcus expression of CPS has only been described in A. viridans var. homari, the causative agent of the lobster disease gaffkemia. The study were studying the relationship between bacterial virulence and CPS thickness in a virulent and avirulent $A$. viridans var. homari strain [24]. In our study, the majority of A. sanguinicola and A. urinae CPS gene homologs were described in genomes of $S$. aureus ssp. aureus MW2 (cap8 genes) and S. pneumoniae TIGR4 (cps4 genes), which are two well-known CPS expressing bacterial species [63-65].

Skov Sørensen et al. investigated expression of CPS of S. pneumoniae and mitis group streptococci [66]. Previously, it was assumed that CPS expression does not take place in commensal organisms as mitis group streptococci. Surprisingly, in a high number of the commensal mitis group streptococci, both the presence of CPS loci and subsequent CPS expression were observed [66]. Based on these results and identification of VFDB gene homologs associated with CPS, we were analyzing how these genes were dispersed within each of the $A$. urinae and $A$. sanguinicola genomes. Very surprisingly, we were identifying putative CPS loci in all the WGS genomes with high certainties of being a real CPS loci due to a number of findings. First, all $A$. sanguinicola and $A$. urinae CPS loci were divided into a highly common (regulatory part) and variable region (CPS biosynthesis) [67,68], as seen with CPS loci of S. agalactiae [69] and S. pneumoniae strains [66]. In S. agalactiae strains, the regulatory function of the common region was, among others, demonstrated with a functional knock-out mutation analysis in which the common region regulated CPS expression and its finetuning [70].

Secondly, CPS gene homologs of the variable region of $A$. sanguinicola and $A$. urinae CPS loci were encoding cell surface polysaccharide biosynthesis proteins as glycosyl transferases, epimerases, and dehydrogenases, which was in line with CPS genes of the variable region of streptococcal and staphylococcal CPS loci. Skov Sørensen et al. [66] and O'Riordan \& Lee [71] described the structural organization of streptococcal and S. aureus CPS locus organization, which consisted of polymerases, epimerases, flippases, dehydrogenases, and sugar transferases such as glycosyl transferase. 
659 Thirdly, A. urinae CPS loci showed structural variations with different CPS locus sizes, genetic content, and 660 organization genetic. The observed genetic CPS loci diversity as five different CPS loci types, mainly 661 separated the 1984-2004 A. urinae CPS loci from the highly diverse 2010-2015 A. urinae CPS loci. This type of 662 structural complexity and organization of CPS genes were also shown within S. pneumoniae [68], S. aureus 663 [71], and Klebsiella ssp. [72] CPS loci.

664 665 666 667 668 669 670 671 672 673 674

\subsubsection{Bacterial adherence.}

In this study, the presence of core-genes that were homologs to genes linked to bacterial adherence of $A$. sanguinicola ( $h t p B, f b p A, I m b$, and $i l p A)$ and $A$. urinae ( $h t p B$, lap, Imb, fbp54, and ilpA) indicates adhesion as an important virulence factor within strains causing UTIs, bacteremia, and IE.

These genes were homologs to FbpA of L. monocytogenes [73] and Fbp54 of S. pyogenes [17], Lmb of S. agalactiae [19], and IIpA of Vibrio vulnificus [74]. The importance of these genes have been demonstrated with reduced adhesion using mutants due to no expression of fibronectin-binding proteins ( $L$. monocytogenes FbpA [73] and S. pyogenes Fbp54 [17]), poor adhesion to immobilized placental laminin and subsequent reduced invasiveness (S. agalactiae Lmb) $[19,75]$, and decreased adhesion to intestinal cells and reduced mortality in mice models (V. vulnificus IIpA) [74,76].

The Listeria adhesion protein LAP is an essential adhesion factor $[20,77]$, which has been demonstrated as a cell surface protein [78,79], and binds Hsp60 [80]. A lap-deficient L. monocytogenes showed reduced adherence and unable to translocate into intestinal cells $[77,80]$. Hsp60 associated cell adherence was also described for Clostridium difficile [81]. In genus Aerococcus, upregulated Hsp60 expression was previously described in A. viridans var. homari [25]. In the current study, both Aerococcus species were having a Hsp60 encoding $h t p B$ gene homolog, whereas only a lap gene homolog in $A$. urinae strains. The presence of lap gene and $h t p B$ gene homologs within $A$. urinae genomes enhances the need for further enlightening of a putative bacterial adherence interaction between these two gene products.

In Gram-positive bacteria, a cell surface exposure of bacterial adhesion proteins can be achieved through a signal peptide sequence and a LPXTG containing cell wall anchoring protein domain [82]. A new class of anchorless and surface exposed Gram-positive proteins lacks the signal peptide and/or the LPXTG motif [82]. In the current study, no adhesion associated gene homologs contained a LPXTG anchoring motif and only A. sanguinicola and $A$. urinae $\mathrm{Lmb}$ and IIpA homolog protein coding genes consisted of a signal peptide sequence, which was in line with the laminin-binding protein Lmb of S. agalactiae [19] and Lbp of S. pyogenes [83], and with the IIpA protein of $V$. vulnificus $[74,76]$.

Neither the A. sanguinicola nor A. urinae gene homologs of fibronectin-binding proteins, the LAP protein, or the Hsp60 (HtpB) proteins contained a signal sequence nor the LPXTG motif. This was indeed in line with 
692

693

694

695

696

697

698

699

700

701

702

703

704

705

706

707

708

709

710

711

712

713

714

715

716

717

718

719

720

721

other atypical and surface exposed adhesion proteins that binds fibronectin (FbpA of L. monocytogenes [73], FbpA of Streptococcus gordonii [84], and PavA of S. pneumoniae [85]), the Listeria adhesion protein LAP of L. monocytogenes [79], and heat shock proteins (Hsp60 of Legionella pneumophila [86] and C. difficile [81]).

\subsubsection{Biofilm formation.}

Only A. sanguinicola strains contained a biofilm associated transcriptional regulator gene homolog (bopD) with low sequence identities. The bopD gene of $E$. faecalis is one out of four bopABCD genes associated with biofilm formation $[87,88]$. We find it questionable whether the A. sanguinicola bopD gene homolog is a biofilm associated gene or simply a transcriptional regulator gene, since the bopABCD locus also contains three other genes. As in vitro biofilm production previously was observed in A. sanguinicola [22] and A. urinae strains [21], the search for gene homologs associated with biofilm production may be a key step to increase the bacterial pathogenicity understanding.

\section{Future perspectives.}

With the development of sequencing technologies and the presence of genomes from pathogenic bacteria, a broad range of analyses for a better understanding of bacterial pathogenicity are facilitated. More attention can be subjected to $A$. sanguinicola and $A$. urinae pathogenicity in order to further step into how these clinical strains may cause infections as UTIs, bacteremia, and IE.

Experimental animal models could be one way to analyze the current pathogenic status of recent 2010-2015 A. urinae strains compared to 1984-2004 strains and how the bacterial pathogenicity and host adaptation may have evolved after the first time period of strain collections. Inclusion of more clinical strains, from even broader time periods, and from geographical different locations are needed to extend these analysis. This also in regards to demonstrate if CPS expression takes place, even though both species only were considered as low pathogenic. The functional meaning of gene homologs which were associated with bacterial adhesion needs to be verified and to reveal if the expressed gene products were bacterial cell surface exposed to maintain the adherence function.

Introduction of WGS in clinical laboratories will illuminate the fully genomic repertoire of these strains and enhance the clinical importance of these strains, including identification of the natural habitat of these bacterial species. 


\section{CONCLUSIONS}

723 This is the first study dealing with comparative WGS analysis of clinical and type strain genomes of $A$. 724 sanguinicola and $A$. urinae. High degree of genomic clustering was observed for strains of $A$. sanguinicola 725 and marked differences within genomes of $A$. urinae strains with regards to the average genome sizes, 726 number and sequence identity of core-genes, proteome conservations, genomic clustering, and phylogenetic 727 analysis.

728 Gene homologs associated with antiphagocytosis and bacterial adherence were identified and putative CPS 729 loci were identified within both species.

730 These findings contributes with novel genetic information of $A$. sanguinicola and $A$. urinae strains which 731 provides an important basis for future understanding of UTIs, bacteremia, and IE pathogenicity caused by 732 these two Aerococcus species. 


\section{COMPETING INTERESTS}

735 The authors declare no competing interest.

\section{FUNDING}

738 The work was supported by the Region Zealand Research Unit (13-000835); The Foundation of Director 739 Jacob Madsen and Spouse Olga Madsen (May 2014); The Foundation of Kurt Bønnelycke and Spouse Mrs 740 Grethe Bønnelycke (10053030); The Council of Research in Region Zealand Næstved/Slagelse/Ringsted 741 Hospitals (December 2013 and October 2014); The Foundation of Helge Peetz and Verner Peetz \& Spouse 742 Vilma Peetz (December 2013); The Region Zealand Foundation for Health Research (12-000095); The

743 Common Research Foundation of Region Zealand and Roskilde University (November 2013); and The Danish 744 Heart Foundation (15-R99-A6040-22951).

\section{ACKNOWLEDGMENTS}

747 Thanks to medical laboratory technicians at the Department of Clinical Microbiology, Slagelse Hospital, 748 Denmark for collaboration during collection and identification of clinical strains. Thanks to Ulrik Stenz 749 Justesen (Department of Clinical Microbiology, Odense University Hospital, Odense, Denmark) for providing 750 clinical strains; and thanks to Elvira Chapka (Department of Microbiology \& Infection Control, Statens Serum 751 Institut, Denmark) for technical assistance in the WGS process.

752

\section{AUTHORS' CONTRIBUTIONS}

754 DC, XCN, and JJC designed the overall study. RD, PSA, MS, and Elvira Chapka contributed to the laboratory 755 work and WGS process. XCN, JJC and SR guided the bioinformatic analysis and DC and KH performed the 756 bioinformatic data analysis. DC wrote the manuscript and all authors contributed to the critical reading. 


\section{REFERENCES}

[1] R.E.O. Williams, A. Hirch, S.T. Cowan, Aerococcus, a New Bacterial Genus, J. Gen. Microbiol. 8 (1953) $475-480$. doi:10.1099/00221287-8-3-475.

[2] M. Tohno, M. Kitahara, S. Matsuyama, K. Kimura, M. Ohkuma, K. Tajima, Aerococcus vaginalis sp. nov., isolated from the vaginal mucosa of a beef cow, and emended descriptions of Aerococcus suis, Aerococcus viridans, Aerococcus urinaeequi, Aerococcus urinaehominis, Aerococcus urinae, Aerococcus christensenii and Aerococcus sa, Int. J. Syst. Evol. Microbiol. 64 (2014) 1229-1236. doi:10.1099/ijs.0.058081-0.

[3] J.J. Christensen, B. Korner, H. Kjaergaard, Aerococcus -like organism - an unnoticed urinary tract pathogen, APMIS. 97 (1989) 539-546. doi:10.1111/j.1699-0463.1989.tb00828.x.

[4] M. Aguirre, M.D. Collins, Phylogenetic analysis of some Aerococcus-like organisms from urinary tract infections: description of Aerococcus urinae sp. nov., J. Gen. Microbiol. 138 (1992) 401-405. doi:10.1099/00221287-138-2401.

[5] P.A. Lawson, E. Falsen, K. Truberg-Jensen, M.D. Collins, Aerococcus sanguicola sp. nov., isolated from a human clinical source, Int. J. Syst. Evol. Microbiol. 51 (2001) 475-479. doi:10.1099/00207713-51-2-475.

[6] J.J. Christensen, I.P. Jensen, J. Faerk, B. Kristensen, R. Skov, B. Korner, the D.A.S. Group, Bacteremia/Septicemia Due to Aerococcus-Like Organisms: Report of Seventeen Cases, Clin. Infect. Dis. 21 (1995) 943-947. doi:10.1093/clinids/21.4.943.

[7] M. Rasmussen, Aerococci and aerococcal infections, J. Infect. 66 (2013) 467-474. doi:10.1016/j.jinf.2012.12.006

[8] Y.N. Guilarte, R. Tinguely, A. Lupo, A. Endimiani, Prevalence and characteristics of fluoroquinolone-resistant Aerococcus urinae isolates detected in Switzerland, Int. J. Antimicrob. Agents. 43 (2014) 474-483. doi:10.1016/j.ijantimicag.2014.01.022.

[9] M. Rasmussen, Aerococcus: an increasingly acknowledged human pathogen, Clin. Microbiol. Infect. 22 (2016) 22-27. doi:10.1016/j.cmi.2015.09.026.

[10] J.J. Christensen, M. Kilian, V. Fussing, K. Andresen, J. Blom, B. Korner, A.G. Steigerwalt, Aerococcus urinae: Polyphasic characterization of the species, APMIS. 113 (2005) 517-525. doi:10.1111/j.16000463.2005.apm_183.x.

[11] E. Senneby, L. Göransson, S. Weiber, M. Rasmussen, A population-based study of aerococcal bacteraemia in the MALDI-TOF MS-era, Eur. J. Clin. Microbiol. Infect. Dis. 35 (2016) 755-762. doi:10.1007/s10096-016-2594-z.

[12] J.J. Christensen, R. Dargis, M. Hammer, U.S. Justesen, X.C. Nielsen, M. Kemp, T.D.M.-T.M.S. Group, Matrixassisted laser desorption ionization-time of flight mass spectrometry analysis of gram-positive, catalasenegative cocci not belonging to the Streptococcus or Enterococcus genus and benefits of database extension, J. Clin. Microbiol. 50 (2012) 1787-1791. doi:10.1128/JCM.06339-11.

[13] O. Opota, G. Prod'hom, C. Andreutti-Zaugg, M. Dessauges, L. Merz, G. Greub, J.P. Chave, K. Jaton, Diagnosis of Aerococcus urinae infections: Importance of matrix-assisted laser desorption ionization time-of-flight mass spectrometry and broad-range 16S rDNA PCR, Clin. Microbiol. Infect. 22 (2016) e1-e2. doi:10.1016/j.cmi.2015.08.026.

[14] X.C. Nielsen, D. Carkaci, R. Dargis, L. Hannecke, J.U. Stenz, M. Kemp, M. Hammer, J.J. Christensen, 16S-23S Intergenic Spacer (ITS) Region Sequence Analysis: Applicability and Usefulness in Identifying Genera and Species Resembling Non-Hemolytic Streptococci, Clin. Microbiol. Open Access. 2 (2013) 1-8. doi:10.4172/23275073.1000130 .

[15] A.L. Flores-Mireles, J.N. Walker, M. Caparon, S.J. Hultgren, Urinary tract infections: epidemiology, mechanisms of infection and treatment options, Nat. Rev. Microbiol. 13 (2015) 269-284. doi:10.1038/nrmicro3432.

[16] M.A. Kielhofner, R.J. Hamill, Role of Adherence in Infective Endocarditis, Texas Hear. Inst. J. 16 (1989) $239-249$. 
802 [17] H.S. Courtney, Y. Li, J.B. Dale, D.L. Hasty, Cloning, sequencing, and expression of a fibronectin/fibrinogenbinding protein from group A streptococci, Infect. Immun. 62 (1994) 3937-3946.

[18] A. Osanai, L. Sheng-Jun, K. Asano, H. Sashinami, D.L. Hu, A. Nakane, Fibronectin-binding protein, FbpA, is the adhesin responsible for pathogenesis of Listeria monocytogenes infection, Microbiol. Immunol. 57 (2013) 253262. doi:10.1111/1348-0421.12030.

[19] B. Spellerberg, E. Rozdzinski, S. Martin, J. Weber-Heynemann, N. Schnitzler, R. Lütticken, A. Podbielski, Lmb, a protein with similarities to the Lral adhesin family, mediates attachment of Streptococcus agalactiae to human laminin, Infect. Immun. 67 (1999) 871-878.

[20] K.M. Burkholder, A.K. Bhunia, Listeria monocytogenes uses Listeria adhesion protein (LAP) to promote bacterial transepithelial translocation and induces expression of LAP receptor Hsp60, Infect. Immun. 78 (2010) 50625073. doi:10.1128/IAI.00516-10.

[21] O. Shannon, M. Mörgelin, M. Rasmussen, Platelet activation and biofilm formation by Aerococcus urinae, an endocarditis-causing pathogen, Infect. Immun. 78 (2010) 4268-4275. doi:10.1128/IAI.00469-10.

[22] E. Senneby, B. Eriksson, E. Fagerholm, M. Rasmussen, Bacteremia with Aerococcus sanguinicola: Case Series with Characterization of Virulence Properties, Open Forum Infect. Dis. 1 (2014) 1-6. doi:10.1093/ofid/ofu025.

[23] I.S. Roberts, The biochemistry and genetics of capsular polysaccharide production in bacteria, Annu. Rev. Microbiol. 50 (1996) 285-315. doi:10.1146/annurev.micro.50.1.285.

[32] A. Gurevich, V. Saveliev, N. Vyahhi, G. Tesler, QUAST: quality assessment tool for genome assemblies,

[24] K.F. Clark, D. Wadowska, S.J. Greenwood, Aerococcus viridans var. homari: The presence of capsule and the relationship to virulence in American lobster (Homarus americanus), J. Invertebr. Pathol. 133 (2016) 20-26. doi:10.1016/j.jip.2015.11.007.

[25] K.F. Clark, S.J. Greenwood, Aerococcus viridans expression of Cpn60 is associated with virulence during infection of the American lobster, Homarus americanus Milne Edwards, J. Fish Dis. 34 (2011) 831-843. doi:10.1111/j.1365-2761.2011.01300.x.

[26] J.J. Christensen, A.M. Whitney, L.M. Teixeira, A.G. Steigerwalt, R.R. Facklam, B. Korner, D.J. Brenner, Aerococcus urinae: Intraspecies Genetic and Phenotypic Relatedness, Int. J. Syst. Bacteriol. 47 (1997) $28-32$. doi:10.1099/00207713-47-1-28.

[27] R.J. Olsen, S.W. Long, J.M. Musser, Bacterial Genomics in Infectious Disease and the Clinical Pathology Laboratory, Arch. Pathol. Lab. Med. 136 (2012) 1414-1422. doi:10.5858/arpa.2012-0025-RA.

[28] D. Carkaci, R. Dargis, X.C. Nielsen, O. Skovgaard, K. Fuursted, J.J. Christensen, Complete Genome Sequences of Aerococcus christensenii CCUG 28831T, Aerococcus sanguinicola CCUG 43001T, Aerococcus urinae CCUG 36881T, Aerococcus urinaeequi CCUG 28094T, Aerococcus urinaehominis CCUG 42038 BT, and Aerococcus viridans CCUG 4311T, Genome Announc. 4 (2016) 1-2. doi:10.1128/genomeA.00302-16.

[29] S. Andrews, FastQC: a quality control tool for high throughput sequence data, www.bioinformatics.babraham.ac.uk/projects/fastqc, (2010).

[30] R. Schmieder, R. Edwards, Quality control and preprocessing of metagenomic datasets, Bioinformatics. 27 (2011) 863-864. doi:10.1093/bioinformatics/btr026.

[31] A. Bankevich, S. Nurk, D. Antipov, A.A. Gurevich, M. Dvorkin, A.S. Kulikov, V.M. Lesin, S.I. Nikolenko, S. Pham, A.D. Prjibelski, A. V. Pyshkin, A. V. Sirotkin, N. Vyahhi, G. Tesler, M.A. Alekseyev, P.A. Pevzner, SPAdes: A New Genome Assembly Algorithm and Its Applications to Single-Cell Sequencing, J. Comput. Biol. 19 (2012) 455-477. doi:10.1089/cmb.2012.0021.

[33] M. V Larsen, S. Cosentino, O. Lukjancenko, D. Saputra, S. Rasmussen, H. Hasman, T. Sicheritz-Pontén, F.M. Aarestrup, D.W. Ussery, O. Lund, Benchmarking of methods for genomic taxonomy, J. Clin. Microbiol. 52 (2014) 
[34] S.F. Altschul, W. Gish, W. Miller, E.W. Myers, D.J. Lipman, Basic local alignment search tool, J. Mol. Biol. 215 (1990) 403-410. doi:10.1016/S0022-2836(05)80360-2.

[35] O. Lukjancenko, M.C. Thomsen, M.V. Larsen, D.W. Ussery, PanFunPro: PAN-genome analysis based on FUNctional PROfiles, F1000Research. 2 (2013) 1-13. doi:10.12688/f1000research.2-265.v1.

[36] D. Hyatt, G.-L. Chen, P.F. LoCascio, M.L. Land, F.W. Larimer, L.J. Hauser, Prodigal: prokaryotic gene recognition and translation initiation site identification, BMC Bioinformatics. 11 (2010) 1-11. doi:10.1186/1471-2105-11119.

[37] E.M. Zdobnov, R. Apweiler, InterProScan - an integration platform for the signature-recognition methods in InterPro, Bioinformatics. 17 (2001) 847-848. doi:10.1093/bioinformatics/17.9.847.

[38] M. Punta, P.C. Coggill, R.Y. Eberhardt, J. Mistry, J. Tate, C. Boursnell, N. Pang, K. Forslund, G. Ceric, J. Clements, A. Heger, L. Holm, E.L.L. Sonnhammer, S.R. Eddy, A. Bateman, R.D. Finn, The Pfam protein families databases, Nucleic Acids Res. 30 (2012) D290-D301. doi:10.1093/nar/gkr1065.

[39] D.H. Haft, J.D. Selengut, O. White, The TIGRFAMs database of protein families, Nucleic Acids Res. 31 (2003) 371-373. doi:10.1093/nar/gkg128.

[40] D. Wilson, R. Pethica, Y. Zhou, C. Talbot, C. Vogel, M. Madera, C. Chothia, J. Gough, SUPERFAMILY Sophisticated comparative genomics, data mining, visualization and phylogeny, Nucleic Acids Res. 37 (2009) D380-D386. doi:10.1093/nar/gkn762.

[41] W. Li, A. Godzik, Cd-hit: A fast program for clustering and comparing large sets of protein or nucleotide sequences, Bioinformatics. 22 (2006) 1658-1659. doi:10.1093/bioinformatics/btl158.

[42] R.C. Edgar, MUSCLE: Multiple sequence alignment with high accuracy and high throughput, Nucleic Acids Res. 32 (2004) 1792-1797. doi:10.1093/nar/gkh340.

[43] D. Darriba, G.L. Taboada, R. Doallo, D. Posada, jModelTest 2: more models, new heuristics and parallel computing, Nat. Methods. 9 (2012) 772. doi:10.1038/nmeth.2109.

[44] S. Guindon, O. Gascuel, A Simple, Fast, and Accurate Method to Estimate Large Phylogenies by Maximum Likelihood, Syst. Biol. 52 (2003) 696-704. doi:10.1080/10635150390235520.

[45] R.S. Kaas, P. Leekitcharoenphon, F.M. Aarestrup, O. Lund, Solving the problem of comparing whole bacterial genomes across different sequencing platforms, PLoS One. 9 (2014) 1-8. doi:10.1371/journal.pone.0104984.

[46] L. Chen, D. Zheng, B. Liu, J. Yang, Q. Jin, VFDB 2016: hierarchical and refined dataset for big data analysis-10 years on., Nucleic Acids Res. 44 (2016) D694-D697. doi:10.1093/nar/gkv1239.

[47] M. Kearse, R. Moir, A. Wilson, S. Stones-Havas, M. Cheung, S. Sturrock, S. Buxton, A. Cooper, S. Markowitz, C. Duran, T. Thierer, B. Ashton, P. Meintjes, A. Drummond, Geneious Basic: An integrated and extendable desktop software platform for the organization and analysis of sequence data, Bioinformatics. 28 (2012) 1647-1649. doi:10.1093/bioinformatics/bts199.

[48] T.N. Petersen, S. Brunak, G. von Heijne, H. Nielsen, SignalP 4.0: discriminating signal peptides from transmembrane regions, Nat. Methods. 8 (2011) 785-786. doi:10.1038/nmeth.1701.

[49] N.Y. Yu, J.R. Wagner, M.R. Laird, G. Melli, S. Rey, R. Lo, P. Dao, S.C. Sahinalp, M. Ester, L.J. Foster, F.S.L. Brinkman, PSORTb 3.0: Improved protein subcellular localization prediction with refined localization subcategories and predictive capabilities for all prokaryotes, Bioinformatics. 26 (2010) 1608-1615. doi:10.1093/bioinformatics/btq249.

[50] A. Krogh, B. Larsson, G. von Heijne, E.L.L. Sonnhammer, Predicting transmembrane protein topology with a hidden Markov model: application to complete genomes, J. Mol. Biol. 305 (2001) 567-580. doi:10.1006/jmbi.2000.4315. 
[51] T. Tatusova, M. Dicuccio, A. Badretdin, V. Chetvernin, E.P. Nawrocki, L. Zaslavsky, A. Lomsadze, K.D. Pruitt, M. Borodovsky, J. Ostell, NCBI prokaryotic genome annotation pipeline, Nucleic Acids Res. 44 (2016) 6614-6624. doi:10.1093/nar/gkw569.

[52] D.W. Ussery, T.M. Wassenaar, S. Borini, Computing for Comparative Microbial Genomics - Bioinformatics for Microbiologists - Chapter 12, Springer-Verlag London, UK, 2009. doi:10.1007/978-1-84800-255-5_2.

[53] E.J. Feil, Small change: keeping pace with microevolution, Nat. Rev. Microbiol. 2 (2004) 483-495. doi:10.1038/nrmicro904.

[54] B. Segerman, The genetic integrity of bacterial species: the core genome and the accessory genome, two different stories, Front. Cell. Infect. Microbiol. 2 (2012) 1-8. doi:10.3389/fcimb.2012.00116.

[55] K.A. Bliven, A.T. Maurelli, Evolution of Bacterial Pathogens within the Human Host, Microbiol. Spectr. 4 (2016) 1-13. doi:10.1128/microbiolspec.

[56] T. Lefébure, M.J. Stanhope, Evolution of the core and pan-genome of Streptococcus: positive selection, recombination, and genome composition, Genome Biol. 8 (2007) R71.1-R71.17. doi:10.1186/gb-2007-8-5-r71.

[57] Y. Feng, C.J. Chen, L.H. Su, S. Hu, J. Yu, C.H. Chiu, Evolution and pathogenesis of Staphylococcus aureus: Lessons learned from genotyping and comparative genomics, FEMS Microbiol. Rev. 32 (2008) 23-37. doi:10.1111/j.1574-6976.2007.00086.x.

[58] A.P. Rooney, J.L. Swezey, R. Friedman, D.W. Hecht, C.W. Maddox, Analysis of core housekeeping and virulence genes reveals cryptic lineages of Clostridium perfringens that are associated with distinct disease presentations, Genetics. 172 (2006) 2081-2092. doi:10.1534/genetics.105.054601.

[59] M. de Been, W. Van Schaik, L. Cheng, J. Corander, R.J. Willems, Recent recombination events in the core genome are associated with adaptive evolution in Enterococcus faecium, Genome Biol. Evol. 5 (2013) 15241535. doi:10.1093/gbe/evt111.

[60] R.L. Marvig, L.M. Sommer, S. Molin, H.K. Johansen, Convergent evolution and adaptation of Pseudomonas aeruginosa within patients with cystic fibrosis, Nat. Genet. 47 (2015) 57-65. doi:10.1038/ng.3148.

[61] S. Tal, V. Guller, S. Levi, R. Bardenstein, D. Berger, I. Gurevich, A. Gurevich, Profile and prognosis of febrile elderly patients with bacteremic urinary tract infection, J. Infect. 50 (2005) 296-305. doi:10.1016/j.jinf.2004.04.004.

[62] A. McNally, F. Alhashash, M. Collins, A. Alqasim, K. Paszckiewicz, V. Weston, M. Diggle, Genomic analysis of extra-intestinal pathogenic Escherichia coli urosepsis, Clin. Microbiol. Infect. 19 (2013) E328-E334. doi:10.1111/1469-0691.12202.

[63] T.T. Luong, C.Y. Lee, Overproduction of Type 8 Capsular Polysaccharide Augments Staphylococcus aureus Virulence Overproduction of Type 8 Capsular Polysaccharide Augments Staphylococcus aureus Virulence, Infect. Immun. 70 (2002) 3389-3395. doi:10.1128/IAI.70.7.3389-3395.2002.

[64] S. Sau, J. Sun, C.Y. Lee, Molecular characterization and transcriptional analysis of type 8 capsule genes in Staphylococcus aureus, J. Bacteriol. 179 (1997) 1614-1621. doi:10.1128/jb.179.5.1614-1621.1997.

[65] R. Jothi, S. Parthasarathy, K. Ganesan, Comparison of the Virulence Factors and Analysis of Hypothetical Sequences of the Strains TIGR4, D39, G54 and R6 of Streptococcus Pneumoniae, J. Comput. Sci. Syst. Biol. 1 (2008) 103-118. doi:10.4172/jcsb.1000010.

[66] U.B. Skov Sørensen, K. Yao, Y. Yang, H. Tettelin, M. Kilian, Capsular Polysaccharide Expression in Commensal Streptococcus Species: Genetic and Antigenic Similarities to Streptococcus pneumoniae, MBio. 7 (2016) e0184416. doi:10.1128/mBio.01844-16.

[67] W. Zhengsong, J.-R. Zhang, Bacterial Capsules - Chapter 3, in: Mol. Med. Microbiol., 2nd ed., Elsevier Ltd, 2015: pp. 33-53. doi:http://dx.doi.org/10.1016/B978-0-12-397169-2.00003-2. 
[68] S.D. Bentley, D.M. Aanensen, A. Mavroidi, D. Saunders, E. Rabbinowitsch, M. Collins, K. Donohoe, D. Harris, L. Murphy, M.A. Quail, G. Samuel, I.C. Skovsted, M.S. Kaltoft, B. Barrell, P.R. Reeves, J. Parkhill, B.G. Spratt, Genetic analysis of the capsular biosynthetic locus from all 90 pneumococcal serotypes, PLoS Genet. 2 (2006) e31. doi:10.1371/journal.pgen.0020031.

[69] D.O. Chaffin, S.B. Beres, H.H. Yim, C.E. Rubens, The Serotype of Type la and III Group B Streptococci Is Determined by the Polymerase Gene within the Polycistronic Capsule Operon, J. Bacteriol. 182 (2000) 44664477. doi:10.1128/JB.182.16.4466-4477.2000.

[70] C. Toniolo, E. Balducci, M.R. Romano, D. Proietti, I. Ferlenghi, G. Grandi, F. Berti, I.M. Ros, R. Janulczyk, Streptococcus agalactiae capsule polymer length and attachment is determined by the proteins CpsABCD, J. Biol. Chem. 290 (2015) 9521-9532. doi:10.1074/jbc.M114.631499.

[71] K. O’Riordan, J.C. Lee, Staphylococcus aureus Capsular Polysaccharides, Clin. Microbiol. Rev. 17 (2004) $218-234$. doi:10.1128/CMR.17.1.218-234.2004.

[72] Y.-J. Pan, T.-L. Lin, C.-T. Chen, Y.-Y. Chen, P.-F. Hsieh, C.-R. Hsu, M.-C. Wu, J.-T. Wang, Genetic analysis of capsular polysaccharide synthesis gene clusters in 79 capsular types of Klebsiella spp., Sci. Rep. 5 (2015) 15573. doi:10.1038/srep15573.

[73] S. Dramsi, F. Bourdichon, D. Cabanes, M. Lecuit, H. Fsihi, P. Cossart, FbpA, a novel multifunctional Listeria monocytogenes virulence factor, Mol. Microbiol. 53 (2004) 639-649. doi:10.1111/j.1365-2958.2004.04138.x.

[74] K.J. Lee, N.Y. Lee, Y.S. Han, J. Kim, K.H. Lee, S.J. Park, Functional characterization of the IlpA protein of Vibrio vulnificus as an adhesin and its role in bacterial pathogenesis, Infect. Immun. 78 (2010) 2408-2417. doi:10.1128/IAI.01194-09.

[75] T. Tenenbaum, B. Spellerberg, R. Adam, M. Vogel, K.S. Kim, H. Schroten, Streptococcus agalactiae invasion of human brain microvascular endothelial cells is promoted by the laminin-binding protein Lmb, Microbes Infect. 9 (2007) 714-720. doi:10.1016/j.micinf.2007.02.015.

[76] S.Y. Goo, Y.S. Han, W.H. Kim, K.-H. Lee, S.-J. Park, Vibrio vulnificus IlpA-induced cytokine production is mediated by toll-like receptor 2, J. Biol. Chem. 282 (2007) 27647-27658. doi:10.1074/jbc.M701876200.

[77] Z.W. Jaradat, J.L. Wampler, A.K. Bhunia, A Listeria adhesion protein-deficient Listeria monocytogenes strain shows reduced adhesion primarily to intestinal cell lines, Med. Microbiol. Immunol. 192 (2003) 85-91. doi:10.1007/s00430-002-0150-1.

[78] B. Jagadeesan, O.K. Koo, K.P. Kim, K.M. Burkholder, K.K. Mishra, A. Aroonnual, A.K. Bhunia, LAP, an alcohol acetaldehyde dehydrogenase enzyme in Listeria, promotes bacterial adhesion to enterocyte-like Caco-2 cells only in pathogenic species, Microbiology. 156 (2010) 2782-2795. doi:10.1099/mic.0.036509-0.

[79] K.M. Burkholder, K.P. Kim, K.K. Mishra, S. Medina, B.K. Hahm, H. Kim, A.K. Bhunia, Expression of LAP, a SecA2dependent secretory protein, is induced under anaerobic environment, Microbes Infect. 11 (2009) 859-867. doi:10.1016/j.micinf.2009.05.006.

[80] J.L. Wampler, K. Kim, Z. Jaradat, A.K. Bhunia, Heat Shock Protein 60 Acts as a Receptor for the Listeria Adhesion Protein in Caco-2 Cells, Infect. Immun. 72 (2004) 931-936. doi:10.1128/IAI.72.2.931-936.2004.

[81] C. Hennequin, F. Porcheray, A.-J. Waligora-Dupriet, A. Collignon, M. Barc, P. Bourlioux, T. Karjalainen, GroEL (Hsp60) of Clostridium difficile is involved in cell adherence, Microbiology. 147 (2001) 87-96. doi:10.1099/00221287-147-1-87.

[82] G.S. Chhatwal, Anchorless Adhesins and Invasins of Gram-Positive Bacteria: a New Class of Virulence Factors, Trends Microbiol. 10 (2002) 205-208. doi:10.1016/S0966-842X(02)02351-X.

[83] Y. Terao, S. Kawabata, E. Kunitomo, I. Nakagawa, S. Hamada, Novel laminin-binding protein of Streptococcus pyogenes, Lbp, is involved in adhesion to epithelial cells, Infect. Immun. 70 (2002) 993-997. doi:10.1128/IAI.70.2.993-997.2002. 
[84] J. Christie, R. McNab, H.F. Jenkinson, Expression of fibronectin-binding protein FbpA modulates adhesion in Streptococcus gordonii, Microbiology. 148 (2002) 1615-1625. doi:10.1099/00221287-148-6-1615.

[85] A.R. Holmes, R. McNab, K.W. Millsap, M. Rohde, S. Hammerschmidt, J.L. Mawdsley, H.F. Jenkinson, The pavA gene of Streptococcus pneumoniae encodes a fibronectin-binding protein that is essential for virulence, Mol. Microbiol. 41 (2001) 1395-1408. doi:10.1046/j.1365-2958.2001.02610.x.

[86] R.A. Garduño, E. Garduño, P.S. Hoffman, Surface-Associated Hsp60 Chaperonin of Legionella pneumophila Mediates Invasion in a HeLa Cell Model, Infect. Immun. 66 (1998) 4602-4610.

[87] M. Hufnagel, S. Koch, R. Creti, L. Baldassarri, J. Huebner, A Putative Sugar-Binding Transcriptional Regulator in a Novel Gene Locus in Enterococcus faecalis Contributes to Production of Biofilm and Prolonged Bacteremia in Mice, J. Infect. Dis. 189 (2004) 420-430. doi:10.1086/381150.

[88] R. Creti, S. Koch, F. Fabretti, L. Baldassarri, J. Huebner, Enterococcal Colonization of the Gastro-Intestinal Tract: Role of Biofilm and Environmental Oligosaccharides, BMC Microbiol. 6 (2006) 1-8. doi:10.1186/1471-2180-660. 